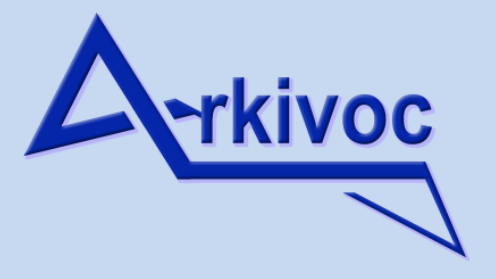

Archive for

Organic Chemistry
The Free Internet Journal

for Organic Chemistry
Review

Arkivoc 2020, part $i, 400-436$

\title{
Orthoesters in heterocycle synthesis
}

\author{
Richard A. Bunce \\ Department of Chemistry, Oklahoma State University, Stillwater, Oklahoma 74078-3071, USA \\ Email: rab@okstate.edu
}

Received 06-17-2020

Accepted 08-25-2020

Published on line 10-17-2020

\section{Abstract}

Orthoesters have occupied an important niche in heterocycle synthesis since the mid- $20^{\text {th }}$ century. They have found wide application in the synthesis of five- and six-membered nitrogen-, oxygen- and sulfur-containing rings from precursors having an acyclic 1,3- or 1,4-arrangement of heteroatoms. Since orthoesters incorporate three geminal alkoxy groups on the central carbon, all of which can be lost, the central carbon of the orthoester $\left[\mathrm{R} \underline{C}(\mathrm{OR})_{3}\right]$ is a synthetic equivalent for "RC $\mathrm{R}^{3+"}$ and can be introduced between the two heteroatoms by means of neutral or acid-catalyzed condensative processes. The current review surveys the literature on the use of orthoesters in heterocycle synthesis and presents numerous examples with mechanisms.

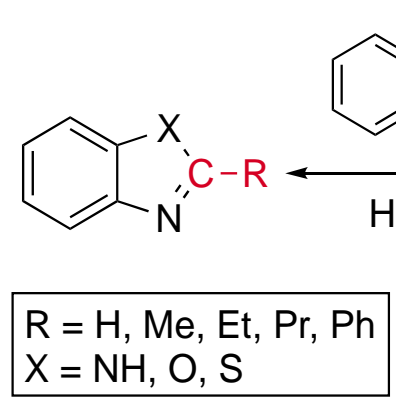<smiles>[R]C(C)OCC</smiles><smiles>[X]C(=O)c1ccccc1N</smiles><smiles>[X]c1nc2ccccc2c(=O)[X]1[H]</smiles><smiles>[R]c1nnc(-c2ccccc2)o1</smiles>

Keywords: Orthoesters, heterocycles, ring formation, condensation, synthesis 


\section{Table of Contents}

1. Introduction

2. Five-Membered Rings

2.1 Monocyclic: two heteroatoms

2.2 Monocyclic: three heteroatoms

2.3 Monocyclic: four heteroatoms

2.4 Fused rings: two heteroatoms

2.5 Fused rings: three heteroatoms

2.6 Complex rings and systems that rearrange

3. Six-Membered Rings

3.1 Monocyclic/fused: two heteroatoms

3.2 Monocyclic/fused: three heteroatoms

3.3 Monocyclic/fused: four heteroatoms

3.4 Complex ring systems

4. Seven-Membered Rings

5. Oxygen Heterocycles

6. Conclusions

Acknowledgments

References

\section{Introduction}

There are many reasons for undertaking studies of synthetic processes involving new reagents. In some cases, a particular target is sought for medicinal evaluation or to prove a natural occurring structure. Alternatively, investigators may wish to develop a new methodology or strategy, or to improve the efficiency, purity or product yield in generating a valuable ring system. Other researchers are interested in testing the scope of a particular reaction or expanding the field by assembling new ring systems with varied substitution patterns which may find applications at a later time. Finally, there are also those chemists whose goal is to probe the mechanistic details of newly discovered transformations.

A number of orthoesters are commercially available, and these are the primary focus in the studies recorded to date. There are several reports detailing syntheses of these simple compounds. ${ }^{1,2}$ The key to future breakthroughs, however, will involve preparing derivatives bearing more functionality on the R group bound to the central carbon. This will permit further elaboration of the structure following installation of the reagent into a heterocyclic framework.

This review seeks to outline some of the uses of orthoesters in heterocycle synthesis. It is arranged according to the size of the ring being formed and the number of heteroatoms within that ring. Beyond this basic outline, each section begins with monocyclic systems and then proceeds to fused rings and finally to complex systems with multiple rings, and occasionally, rearranged products. The review focuses predominantly on reactions where the orthoester is directly installed into the heterocycle. Sequences where an orthoester is used to scavenge water or to prepare an isolable intermediate that is later converted to a heterocycle are generally not included. Most of the studies surveyed targeted nitrogen heterocycles, with some also containing oxygen and sulfur. Finally, several recent efforts have endeavored to prepare heterocycles incorporating only oxygen in the ring, and these are included at the end. 


\section{Five-Membered Rings}

\subsection{Monocyclic: two heteroatoms}

There are relatively few reports describing the synthesis of simple five-membered rings containing only two heteroatoms. However, a study by Hassine and $\mathrm{Kacem}^{3}$ described an approach to enantiopure 2,5disubstituted-3-(phenylamino)-3,5-dihydro-4H-imidazol-4-ones (Scheme 1). Phenylhydrazides 1 were prepared from (L)- $\alpha$-amino acid ester hydrochlorides by treatment with phenylhydrazine in the presence of triethylamine. Heterocyclization with an orthoester (2 equiv) and acetic acid ( $\mathrm{AcOH}, 0.1$ equiv) under neat conditions at $80{ }^{\circ} \mathrm{C}$ led, via imino ether $\mathbf{2}$, to the chiral products $\mathbf{3}$ in $52-81 \%$ yields. Interestingly, in a system which could close to a five- or six-membered ring (3 or $\mathbf{4}$, respectively), the smaller ring was formed by addition of the less basic nitrogen to the carbon of the intermediate imino ether. Compound $\mathbf{3}$ closely resembles structures that have exhibited significant fungicidal properties.

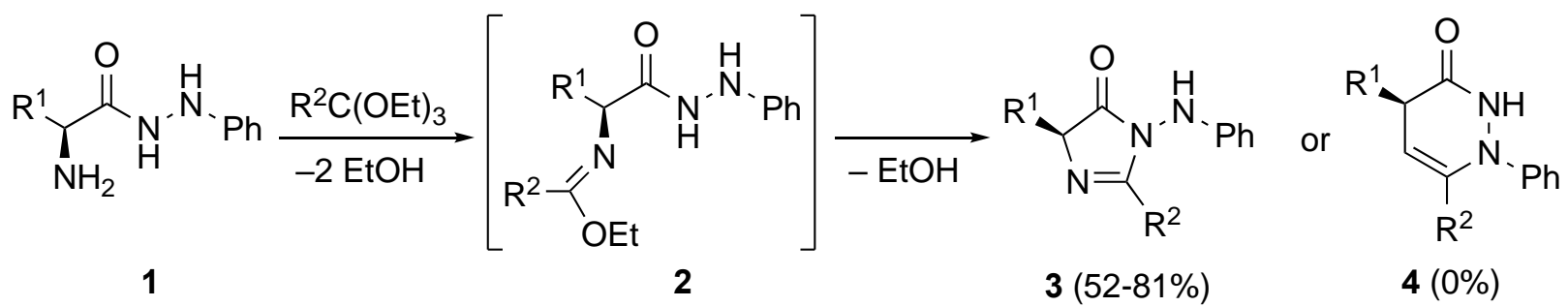

Scheme 1. Synthesis of 3,5-dihydro-4H-imidazol-4-ones from phenylhydrazide.

A family of drug candidates has also been designed to evaluate the interaction of $(4 R S, 5 S R)-4,5-b i s(4-$ hydroxyphenyl)-2-imidazolines with estrogen receptor models to identify potential drugs to treat breast cancer. In this project, Gust and co-workers prepared a series of racemic 4,5-diarylimidazolines. ${ }^{4}$ The preparation of one of the more robust compounds is illustrated in Scheme 2. Treatment of meso diamine 5 with triethyl orthoformate afforded the imidazoline 6 , also meso due to the delocalized $\pi$-bond between the nitrogen atoms. N-alkylation of 6 [1. $n$-BuLi, tetrahydrofuran (THF)-hexane; 2 . Etl, $-78{ }^{\circ} \mathrm{C} \rightarrow 20{ }^{\circ} \mathrm{C}$ ], followed by ether cleavage $\left[\mathrm{BBr}_{3}\right.$, dichloromethane (DCM), $\left.-60{ }^{\circ} \mathrm{C} \rightarrow 20{ }^{\circ} \mathrm{C}\right]$ gave the target compound 7 as a pair of enantiomers in $40 \%$ overall yield.
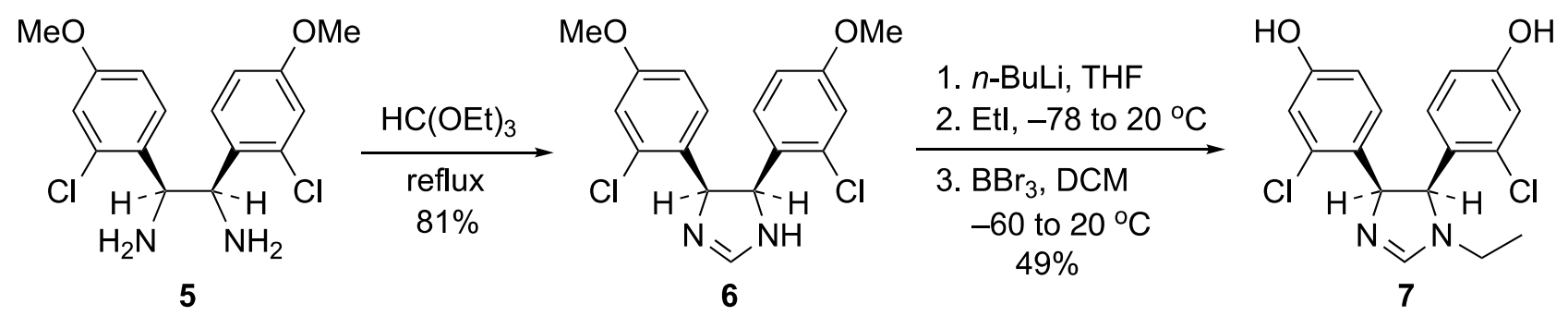

Scheme 2. Synthesis of 4,5-diarylimidazolines from meso diamine 5.

Kim and co-workers ${ }^{5}$ offered an efficient conversion of aroylnitromethanes 8 to 2,5-diaryloxazoles 9 by in situ reduction of the nitro function in the presence of trimethyl orthobenzoate (Scheme 3). The reduction was carried out using indium powder and $\mathrm{AcOH}$ in acetonitrile, and the yields were 45-75\%. Lower recoveries and less pure products were noted when the aryl ring of the substrate was substituted by a halogen or an acid- 
sensitive functional group. Attempts to extend the reaction to non-aromatic orthoesters produced less satisfactory outcomes.

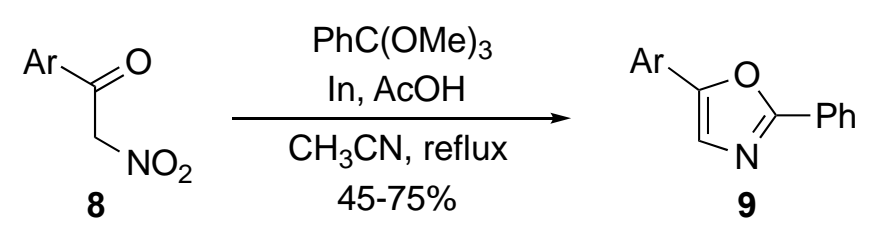

Scheme 3. Synthesis of 2,5-diaryloxazoles from aroylnitromethanes.

\subsection{Monocyclic: three heteroatoms}

Bel'skaya and colleagues ${ }^{6}$ have disclosed an approach to 1,2,3-triazoles from 2-arylhydrazono-2-carbamoylacetamidoximes (Scheme 4). The cyclization substrates 10 were prepared by condensation of hydroxylamine hydrochloride with arylhydrazonocyanoacetamides in the presence of bicarbonate base in $N, N$ dimethylformamide (DMF). Subsequent treatment of 10 with triethyl orthoformate afforded the 1,2,3triazoles by a multistep process. The initial condensation generated 2-arylhydrazono-2-(1,2,4-oxadiazol-4$\mathrm{yl}$ )acetamide 11 by heterocyclization of the amidoxime function with the orthoester. Intermediate $\mathbf{1 1}$ exists as an $E-Z$ equilibrium involving the side chain hydrazone double bond from which the $E$ isomer reacts via 12 and 13 in a Boulton-Katritzky rearrangement to give the triazole 14. The final product $\mathbf{1 4}$ exists as an equilibrium between two tautomeric structures.

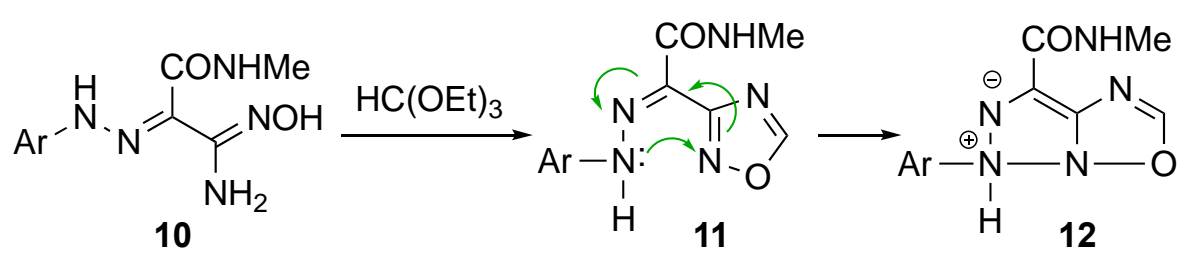<smiles>CNc1nn([Al])nc1C(N)=O</smiles><smiles>C=CC(=O)c1nn([Al])[nH]/c1=N\C=O</smiles>
14

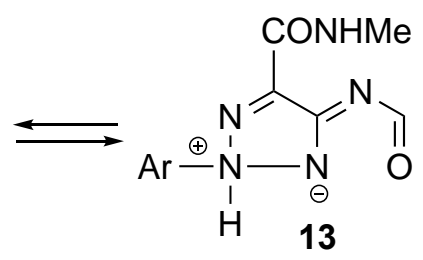

Scheme 4. Synthesis of 1,2,3-triazoles from 2-arylhydrazono-2-carbamoylacetamidoximes.

Classical approaches to oxadiazoles involved dehydrative ring closure of diacylhydrazines by corrosive reagents such as thionyl chloride or phosphorus oxychloride. ${ }^{7}$ Since that time, improvements have been made that allow milder conditions to access these structures. Varma and Polshettiwar ${ }^{8}$ reported the conversion of aromatic acid hydrazides 15 to 1,3,4-oxadiazoles 16 using an orthoester as the solvent with catalytic Nafion ${ }^{\circledR}$ $\mathrm{NR50}$ acid resin under microwave conditions [MW, $300 \mathrm{~W}, 135^{\circ} \mathrm{C}, 1.5 \mathrm{~h}$ ]. These same authors also advanced a synthesis of 1,3,4-thiadiazoles 17 by similar treatment of 15 with an orthoester and $\mathrm{P}_{4} \mathrm{~S}_{10} / \mathrm{Al}_{2} \mathrm{O}_{3}{ }^{9}[300 \mathrm{~W}, 130$ ${ }^{\circ} \mathrm{C}, 25 \mathrm{~min}$ ]. Yields for both methods were 65-90\% (Scheme 5). 


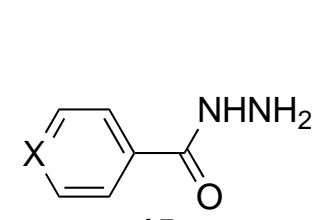

15

$$
\begin{aligned}
& \mathrm{R}=\mathrm{H}, \mathrm{Et}, \mathrm{Ph} \\
& \mathrm{X}=\mathrm{CH}, \mathrm{N}
\end{aligned}
$$
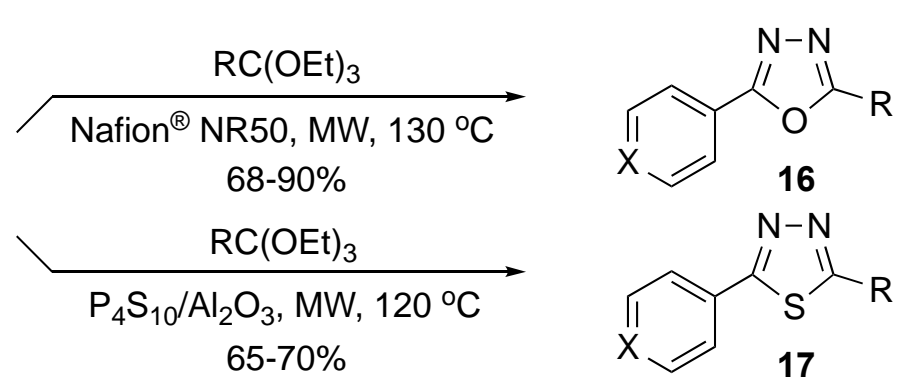

Scheme 5. Synthesis of 1,3,4-oxadiazoles and 1,3,4-thiadiazoles from aromatic acid hydrazides.

A later paper by Bunce and co-workers ${ }^{10}$ employed ammonium chloride as a catalyst in boiling ethanol (EtOH) to convert benzoic hydrazides 18 to 1,3,4-oxadiazoles 22. Most of these reactions were complete within $1 \mathrm{~h}$, except for those employing triethyl orthobenzoate where 2-10 h were required. This procedure was found to require considerably less of the orthoester (1.1-1.2 equiv) compared with earlier methods. Yields were generally $>75 \%$ except for substrates bearing strongly electron-withdrawing substituents. A proposed mechanism for this process is illustrated in Scheme 6. Initial loss of EtOH from the orthoester, and attack by hydrazide 18 on the stabilized carbocation, would give 19. Protonation of 19, followed by loss of a second molecule of EtOH, would yield 20. Cyclization to 21 and elimination of the third molecule of EtOH would then afford oxadiazole 22.

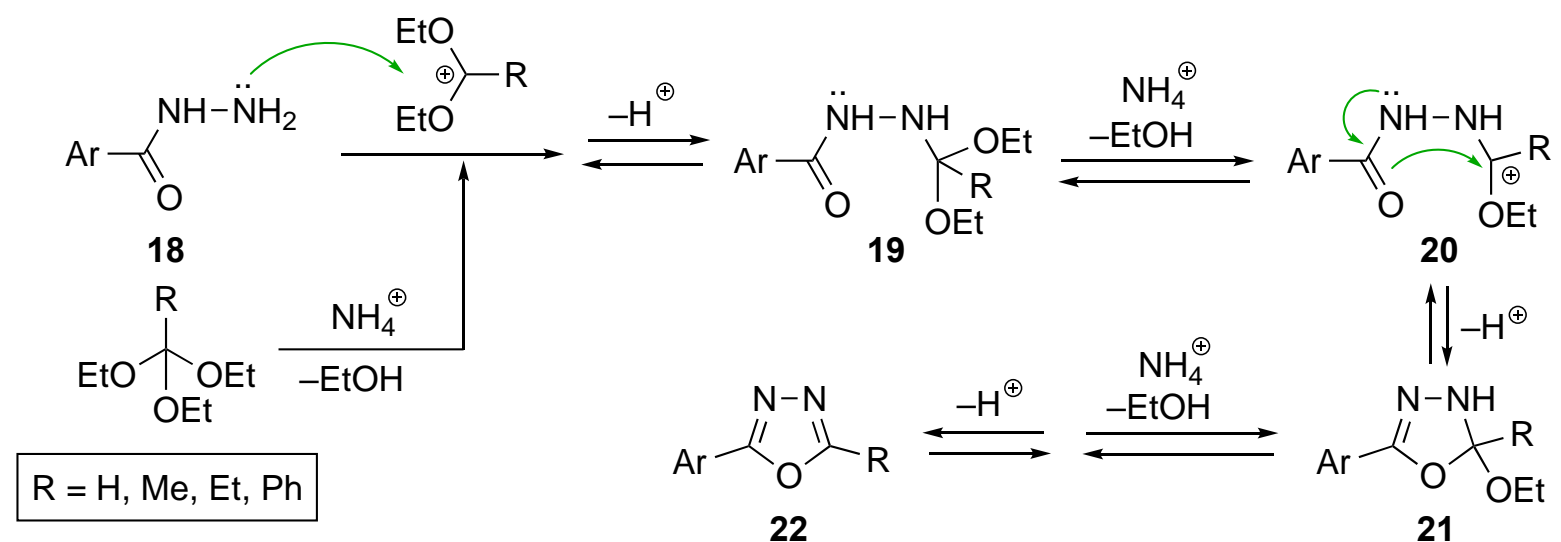

Scheme 6. Synthesis of 1,3,4-oxadiazoles from benzoic hydrazides.

Five mechanistic studies have appeared from the Kudelko group ${ }^{11-15}$ examining the cyclization of hydrazides bearing side chain functionality. 3-(2-Furyl)acrylic or 3-(2-thienyl)acrylic hydrazides reacted normally with a series of triethyl orthoesters in $\mathrm{AcOH}$ to give the expected 2,5-disubstituted 1,3,4oxadiazoles. $^{11}$ In a subsequent report, ${ }^{12}$ however, an $\mathrm{AcOH}$-catalyzed conversion of $\alpha$-mercaptopropionic hydrazide (23) to 1,3,4-oxadiazoles 24 was described. This reaction afforded not only the expected heterocycle but also $4 \mathrm{H}$-1,3,4-thiadiazin-5(6H)-one $\mathbf{2 5}$ derived from participation by the side chain thiol group in the reaction. While thiadiazinone $\mathbf{2 5}$ was the exclusive product from triethyl orthoformate, oxadiazole $\mathbf{2 4}$ was the only product when triethyl orthobenzoate was used (Scheme 7). The orthoacetate and orthopropionate esters afforded mixtures of both products. 


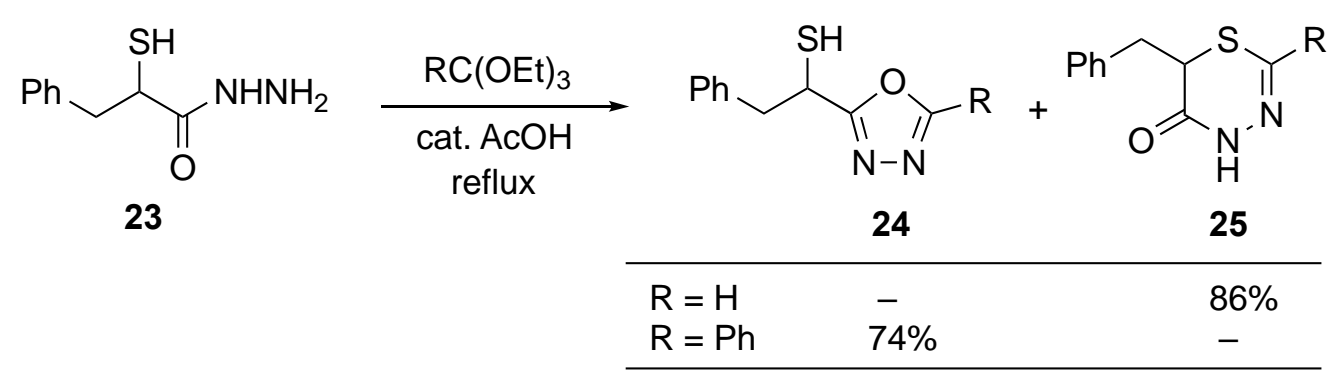

Scheme 7. Reaction of $\alpha$-mercaptopropionic hydrazide with triethyl orthoformate and triethyl orthobenzoate.

A related study $^{13}$ described the reaction of 2-hydroxy-2,2-diphenylacetic hydrazide (26), which incorporated an alcohol substituent $\alpha$ to the hydrazide function. Unlike the sulfur analog 23, the alcohol oxygen did not participate in the reaction to give a $4 H-1,3,4$-oxadiazin-5(6H)-one 27 and a 1,3,4-oxadiazole was not formed. Instead, 1,2,4-triazole $\mathbf{3 0}$ was isolated in $82 \%$ yield (Scheme 8 ). The authors proposed that two molecules of the hydrazide condensed with triethyl orthoacetate via $\mathbf{2 8}$ to form N,N'-bisformamidine 29, which then cyclized to provide the triazole. The structural assignment of $\mathbf{3 0}$ was confirmed by $\mathrm{X}$-ray crystallographic analysis.

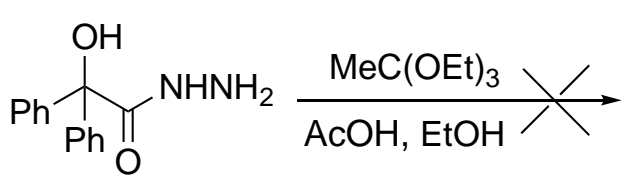

26<smiles>CC1=NNC(=O)C(c2ccccc2)(c2ccccc2)O1</smiles>

27
$\underset{\mathrm{AcOH}, \mathrm{EtOH}}{\stackrel{\mathrm{MeC}(\mathrm{OEt})_{3}}{\longrightarrow}}$

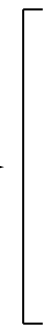<smiles>CCOC(C)=NNC(=O)C(O)(c1ccccc1)c1ccccc1</smiles>

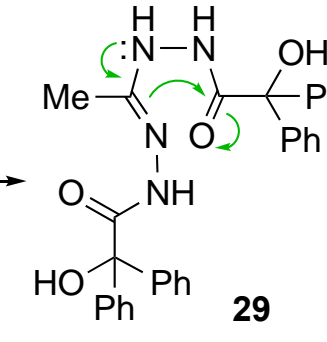

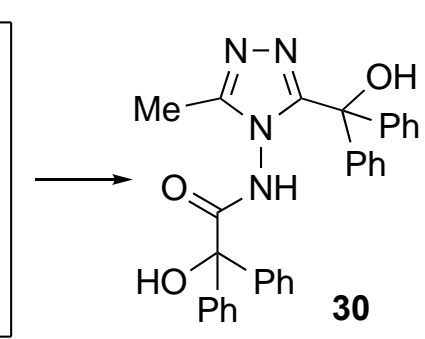

Scheme 8. Reaction of 2-hydroxy-2,2-diphenylacetic hydrazide with triethyl orthoacetate.

A fourth paper reported a synthesis of bis(1,3,4-oxadiazol-2-yl)phenylmethyl sulfides 32 from 1,1'diphenylthiodiacetic acid dihydrazide (31) and several triethyl orthoesters. ${ }^{14}$ This reaction led not only to the desired sulfide-linked heterocycles, but also resulted in cleavage of the carbon-sulfur bond in the substrate with formation of oxadiazoles $\mathbf{3 3}$ and $\mathbf{3 4}$. The results for two of the orthoesters are shown in Scheme 9.

Finally, Kudelko and Zielinski investigated the transformation of chiral N-protected phenylglycine hydrazides 35 to 5-substituted-2-(1-amino-1-phenylmethyl)-1,3,4-oxadiazoles 36 (Scheme 10). ${ }^{15}$ Interestingly, the solvent used for the reaction proved important with respect to the product stereochemistry. Using the relatively polar orthoester as the reaction medium resulted in racemic 1,3,4-oxadiazoles, while nonpolar benzene led to chiral products. Cleavage of the tert-butoxycarbonyl (Boc) protecting group with trimethylsilyl triflate in dry DCM gave the deprotected 1,3,4-oxadiazoles 37 as the triflate salts. It was unclear whether the products of deprotection were chiral, but no optical rotations were given for the final salts. 


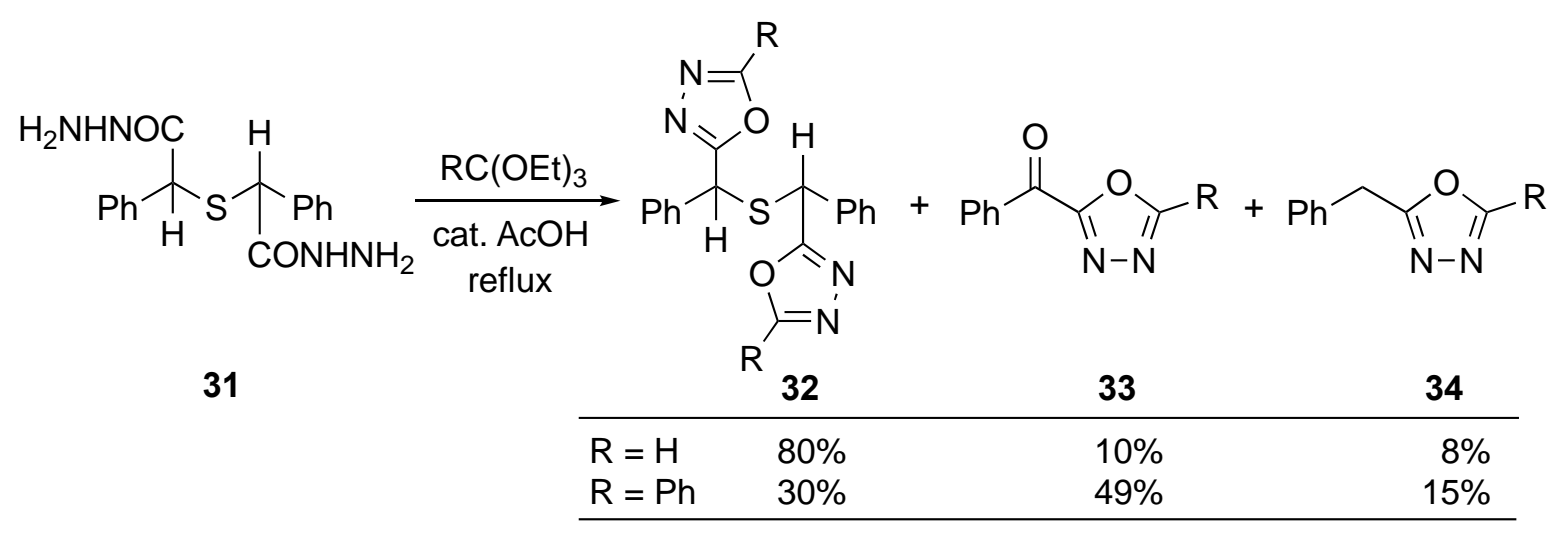

Scheme 9. Reaction of 1,1'-diphenylthiodiacetic acid dihydrazide with two triethyl orthoesters.

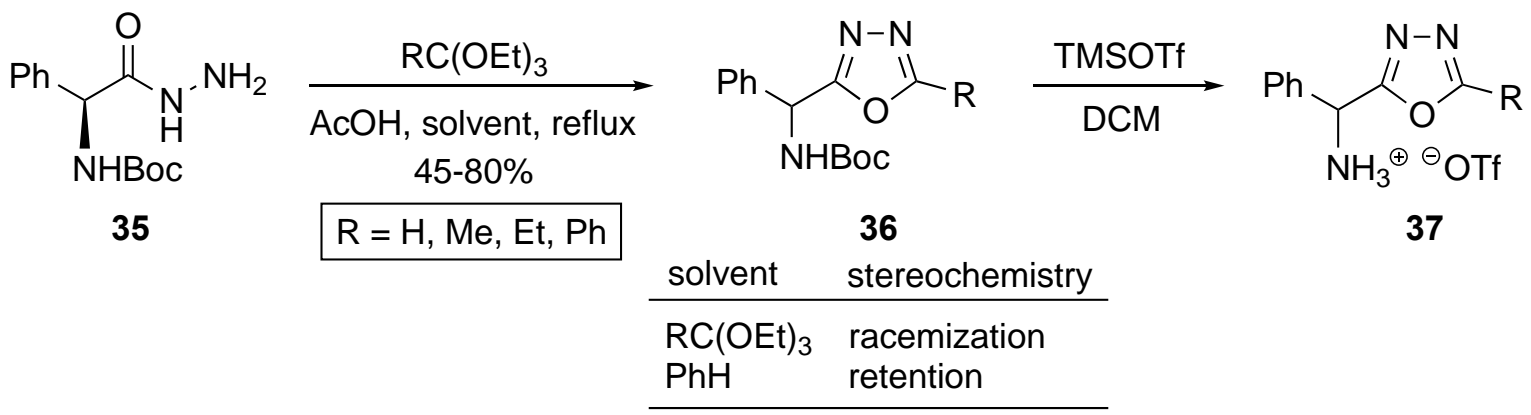

Scheme 10. Reaction of N-protected phenylglycine hydrazides with triethyl orthoesters.

Another investigation by Toche and co-workers ${ }^{16}$ described the synthesis of functionalized $1,3,4$ thiadiazoles from thiosemicarbazides, orthoesters and cyclic or acyclic anhydrides catalyzed by sulfamic acid. Initially, the orthoester condensed with thiosemicarbazide 38 in the presence of sulfamic acid to form 1-(1ethoxyalkylidene)thiosemicarbazide 39, which reacted with the anhydride to form the (5-substituted-1,3,4thiadiazol-2-ylcarbamoyl) aliphatic amide acid derivatives 40 in 68-90\% yield. A representative example using triethyl orthoacetate and succinic anhydride is depicted in Scheme 11 for the conversion of $\mathbf{3 8}$ to $\mathbf{4 0 .}$

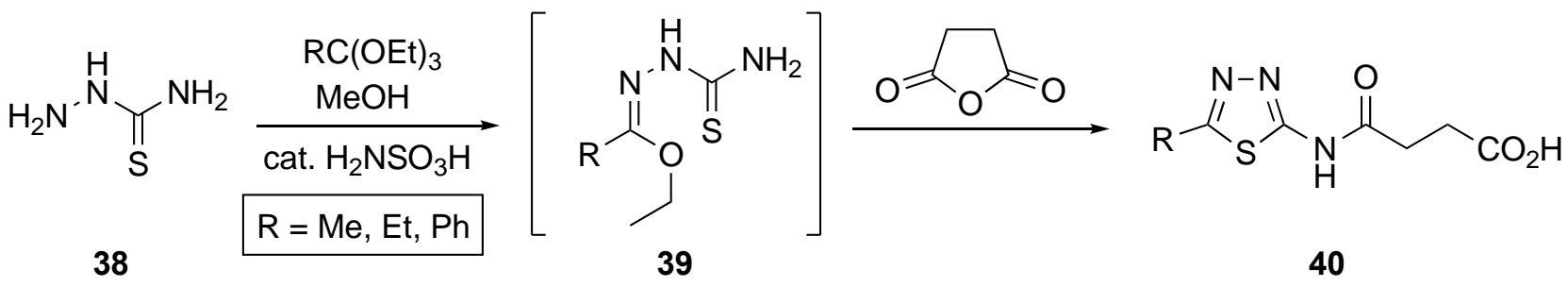

Scheme 11. Synthesis of functionalized thiadiazoles from thiosemicarbazide.

\subsection{Monocyclic: four heteroatoms}

Two studies have enlisted orthoesters for the synthesis of tetrazoles. Tetrazoles are potentially valuable as they can be used in drugs as isosteres of the carboxylic acid function. Isosteres are molecular fragments with similar shapes and electronic properties to other groups that are sometimes introduced into potential drug molecules to modify solubility, toxicity or metabolism. The classic synthesis of these systems requires 
treatment of nitriles with azide salts in polar aprotic solvents. ${ }^{17}$ This strategy involves a concerted 1,3-dipolar addition to the nitrile, while orthoester-based syntheses proceed by stepwise mechanisms.

Aridoss and Laali $^{18}$ contributed a tetrazole synthesis involving reaction of an amine $\mathbf{4 1}$ with trimethylsilyl azide (42) and triethyl orthoformate catalyzed by a Brønsted acid ionic liquid (Scheme 12). In this account, the amines $\mathbf{4 1}$ were generally aniline derivatives, but benzylamine and 2-furylamine were also examined. Best results were obtained using the orthoester as the solvent with a catalytic amount of 1-methyl3-(3-sulfopropyl)- $1 \mathrm{H}$-imidazol-3-ium triflate at $50-60{ }^{\circ} \mathrm{C}$ for $0.5-3 \mathrm{~h}$. Yields were in the range of $77-95 \%$ and did not appear to be sensitive to the electronic nature of substituents on the aromatic rings. Several mechanistic pathways were evaluated and discussed based on density functional theory calculated energies of the possible intermediates.

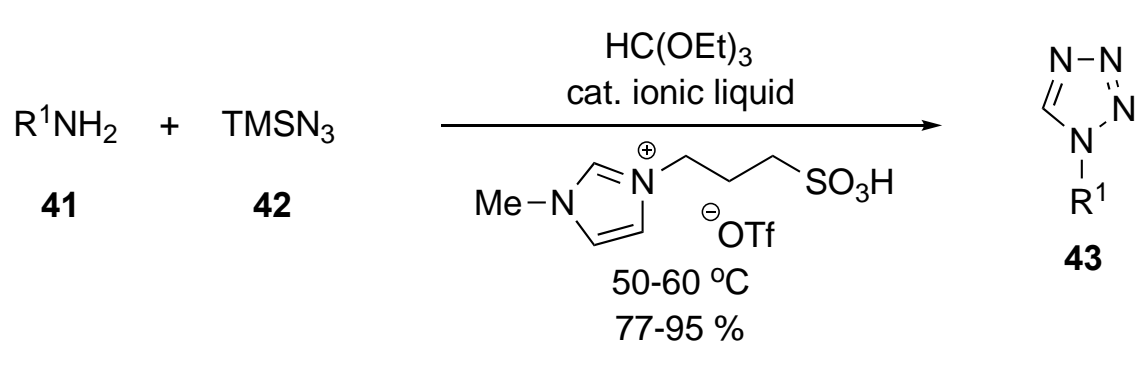

Scheme 12. Synthesis of tetrazoles promoted by a Brønsted acid ionic liquid.

A second study evaluated this same transformation promoted by $\mathrm{AcOH} .{ }^{19}$ The optimum ratio of $\mathrm{RNH}_{2}$ : $\mathrm{HC}(\mathrm{OEt})_{3}: \mathrm{NaN}_{3}: \mathrm{AcOH}$ was 1:3:1.1:8 at 80-100 ${ }^{\circ} \mathrm{C}$ for $2-3 \mathrm{~h}$. Yields were generally greater than $70 \%$ for a wide selection of aliphatic and aromatic amines. The proposed mechanism involved condensation of the aliphatic or aromatic primary amine and triethyl orthoformate to generate imino ether $\mathbf{4 4}$, or amidine $\mathbf{4 5}$, followed by azide addition-elimination to give $\mathbf{4 6}$, which cyclized to tetrazole $\mathbf{4 7}$ (Scheme 13).

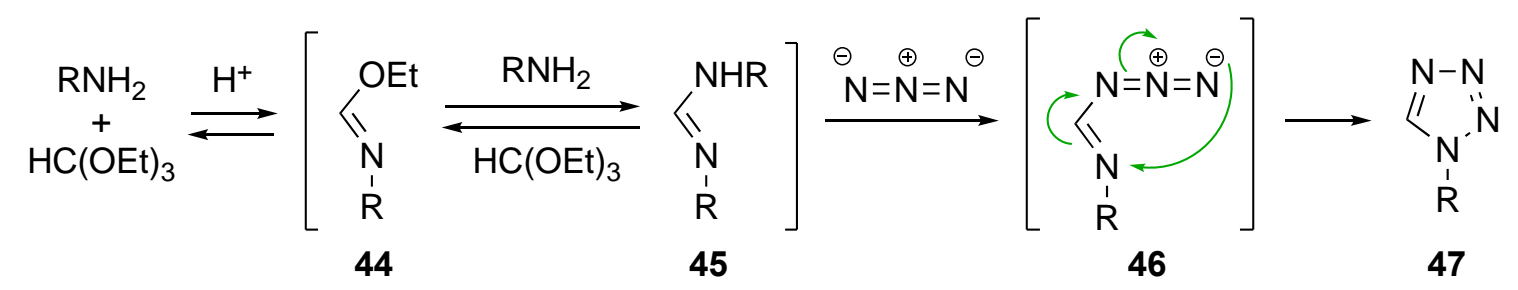

Scheme 13. Synthesis of tetrazoles from amines, sodium azide and triethylorthoformate.

\subsection{Fused rings: two heteroatoms}

An intense effort has been devoted to optimizing protocols for the synthesis of benzimidazoles, benzoxazoles and benzothiazines using orthoesters. Classical routes required dehydrative ring closure of 2-amino-, 2hydroxy- or 2-mercaptoanilides under relatively harsh conditions. ${ }^{20}$ More recently, numerous approaches have employed orthoesters as a source of " $\mathrm{RC}^{3+}$ " to position a carbon between two aryl-bound heteroatoms to form the benzo-fused azole rings. Today, these three scaffolds are considered priviledged ring systems as they are core structures found in many drugs. Over the past 10 years, many different strategies and catalysts have been evaluated and the major contributions are highlighted below.

One of the first reports on the use orthoesters to generate heterocycles was a serendipitous synthesis of benzothiazoles and benzoxazoles that appeared in $1961 .^{21}$ In a study aimed at preparing 2-(N- 
alkylamino)benzenethiols from 2-aminobenzenethiol and ethyl orthoformate, Jenkins and co-workers instead isolated benzothiazole. The method was extended to the synthesis of benzoxazoles as well, with yields for both systems in the $75-85 \%$ range. A later study by Kaboudin et al. applied this strategy to the preparation of benzimidazoles by simple heating of $o$-phenylenediamine derivatives with orthoesters to afford the target heterocycles in $50-85 \% .^{22}$

Although initial investigations utilized no catalyst, subsequent studies focused on the use of different agents to promote the reaction and methods that could be exploited to prepare all three of these benzo-fused heterocycles from commercial orthoesters. Aridoss and Laali ${ }^{18}$ explored the use of Brønsted acid ionic liquids as catalyts under neat conditions with sonication. Villemin and co-workers ${ }^{23}$ utilized montmorillonite KSF clay in refluxing toluene. The Bunce group ${ }^{24}$ employed catalytic ammonium chloride in boiling EtOH to facilitate the process. A mechanism for this last entry is shown in Scheme 14. Initial loss of EtOH from the orthoester and attack by the aromatic amino function of $\mathbf{4 8}$ on the stabilized carbonium ion would give $\mathbf{4 9}$. Protonation of $\mathbf{4 9}$, followed by loss of a second molecule of EtOH, would give $\mathbf{5 0}$. Cyclization of $\mathbf{5 0}$ to $\mathbf{5 1}$ and release of a third molecule of EtOH would then furnish benzazole $\mathbf{5 2}$.

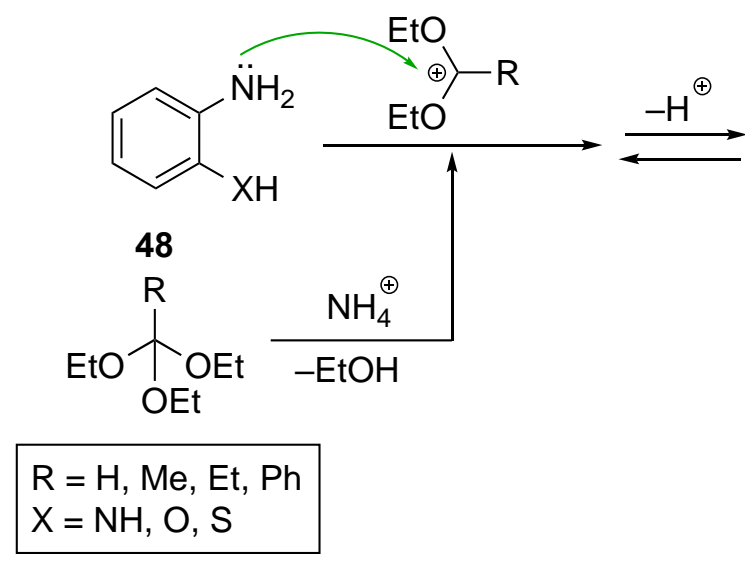<smiles>[X]c1ccccc1NC([R])(OCC)OCC</smiles>

49

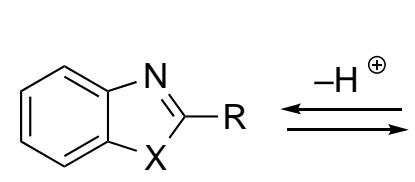

52

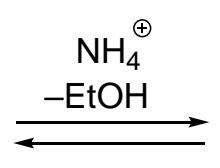<smiles></smiles>

50

$\uparrow \downarrow-H^{\oplus}$

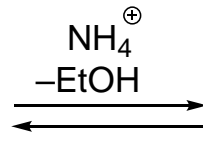<smiles>[X]C1([R])[X]c2ccccc2N1</smiles>

51

Scheme 14. Synthesis of benzazoles promoted by ammonium chloride.

A project in the Jeffries-El group examined functionalized orthesters in a novel preparation of 2,6disubstituted benzobisthiazoles as potential organic semiconductors. ${ }^{25}$ The synthesis involved treatment of 2,5-diamino-1,4-benzenedithiol dihydrochloride (53) with pyridine in $N, N$-dimethylacetamide (DMA). To the resulting solution was added triethyl orthopropiolate $\mathbf{5 4}$ and catalytic yttrium(III) triflate. After stirring at 55 ${ }^{\circ} \mathrm{C}$ for $1 \mathrm{~h}$, the reaction was worked up to deliver 2,6-bis(trimethylsilylethynyl)benzo[1,2-d:4,5- $d$ ] bisthiazole (55) in $38 \%$ yield (Scheme 15$)$.<smiles>Nc1cc(S)c(N)cc1S</smiles>

53

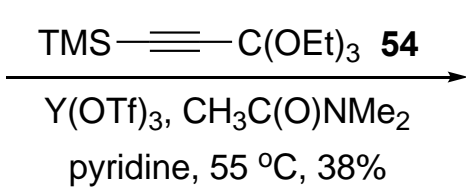

pyridine, $55^{\circ} \mathrm{C}, 38 \%$

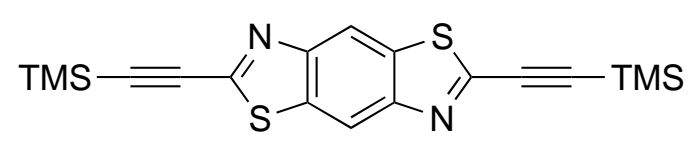

55

Scheme 15. Synthesis of potential bisthiazole organic semiconductors. 
Beyond this, 1,3-dibromo-5,5-dimethylhydantoin ${ }^{26}$ and mildly acidic solvents, such as 1,1,1,3,3,3hexafluoro-2-propanol, ${ }^{27}$ were shown to accelerate the formation of benzimidazoles while Brønsted acids, such as silica-supported tin exchanged silicotungstic acid $^{28}$ and silica sulfuric acid, ${ }^{29}$ were employed to prepare benzoxazoles. Finally, Marko and co-workers ${ }^{30}$ reported the use of boron trifluoride etherate $\left(\mathrm{BF}_{3} \cdot \mathrm{OEt}_{2}\right)$ in $\mathrm{DCM}$ at $23{ }^{\circ} \mathrm{C}$ to promote reactions of complex orthoesters which permitted some interesting post-cyclization transformations such as those shown in Scheme 16. All three benzazoles were prepared and all of the yields were high. Two of these processes are outlined for the conversion of $\mathbf{5 6}$ to $\mathbf{5 9}$ and $\mathbf{6 2}$. This publication also cited references to milder variants of the Pinner reaction to prepare the required orthoesters.

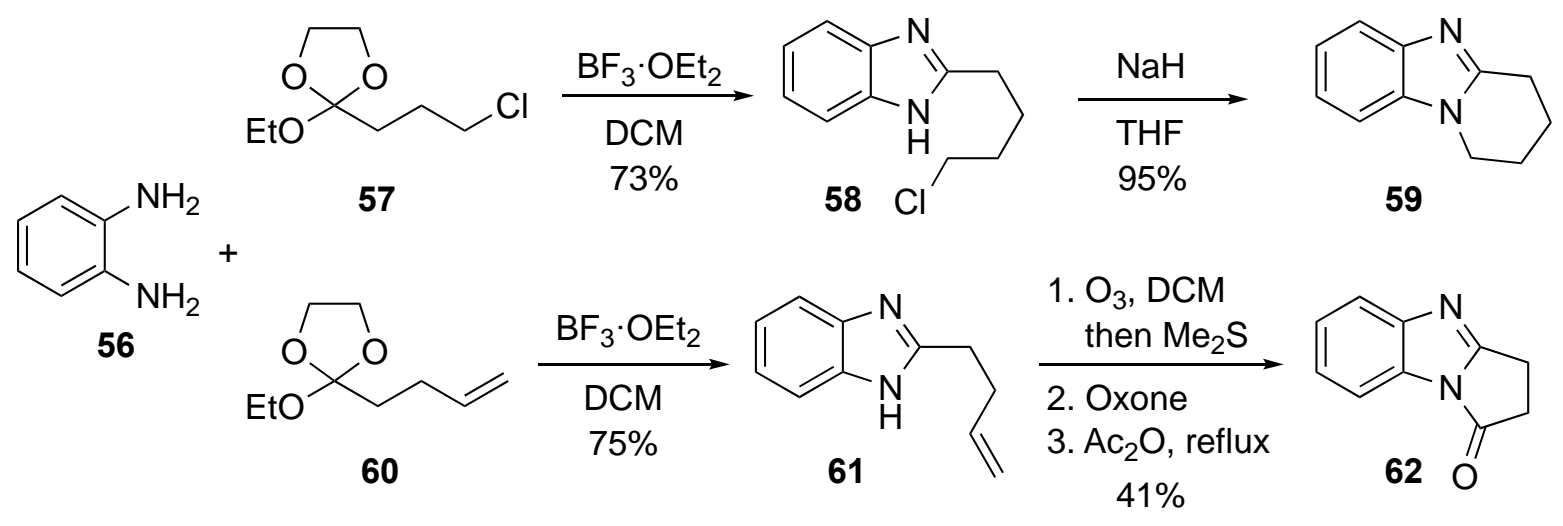

Scheme 16. Formation of benzimidazoles from orthoesters functionalized for post-cyclization reactions.

MW energy has also been used as a means of promoting the synthesis of benzimidazoles as well as more complex fused derivatives. MW-accelerated $\mathrm{S}_{\mathrm{N}} \mathrm{Ar}$ displacement of chloride [300 W, $\left.120{ }^{\circ} \mathrm{C}, 20 \mathrm{~min}\right] \mathrm{from}$ 1-chloro-2-nitro-4-(trifluoromethyl)benzene (63), and catalytic reduction of the nitro group, generated the required 1,2-diamino precursors 64 in 94-98\% yield. Reaction of 64 with a series of orthoesters [300 W, 150$190{ }^{\circ} \mathrm{C}, 30 \mathrm{~min}$ ] then afforded access to a modest library of trifluoromethyl-substituted benzimidazoles 65 (Scheme 17). ${ }^{31}$

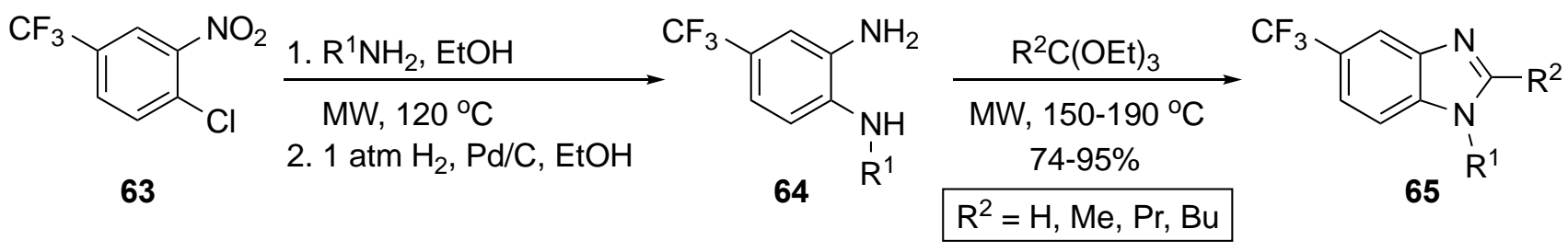

Scheme 17. Synthesis of trifluoromethyl-substituted benzimidazoles.

Two additional studies have appeared wherein the fused heterocycle was generated from an ophenylenediamine or 2-aminophenol created in situ by reduction of the corresponding nitro compound (Scheme 18). The Shen team catalytically reduced 2-nitroanilines 66 in the presence of an orthoester to prepare 1,2-disubstituted benzimidazoles $67 .^{32}$ These authors reported 36 examples with yields in the 45-95\% range. In this sequence, chloroaromatic substrates failed to react cleanly, and this likely resulted from competitive dehalogenation under catalytic reduction with $\mathrm{Pd} / \mathrm{C}$. Kim and co-workers used dissolving metal conditions to reduce 2-nitrophenols 68 with indium metal and $\mathrm{AcOH}$ in benzene to produce 2-substituted 
benzoxazoles $69 .^{5}$ This protocol permitted the preparation of 30 derivatives in $50-98 \%$ yields and aryl-bound halogens presented no problems.

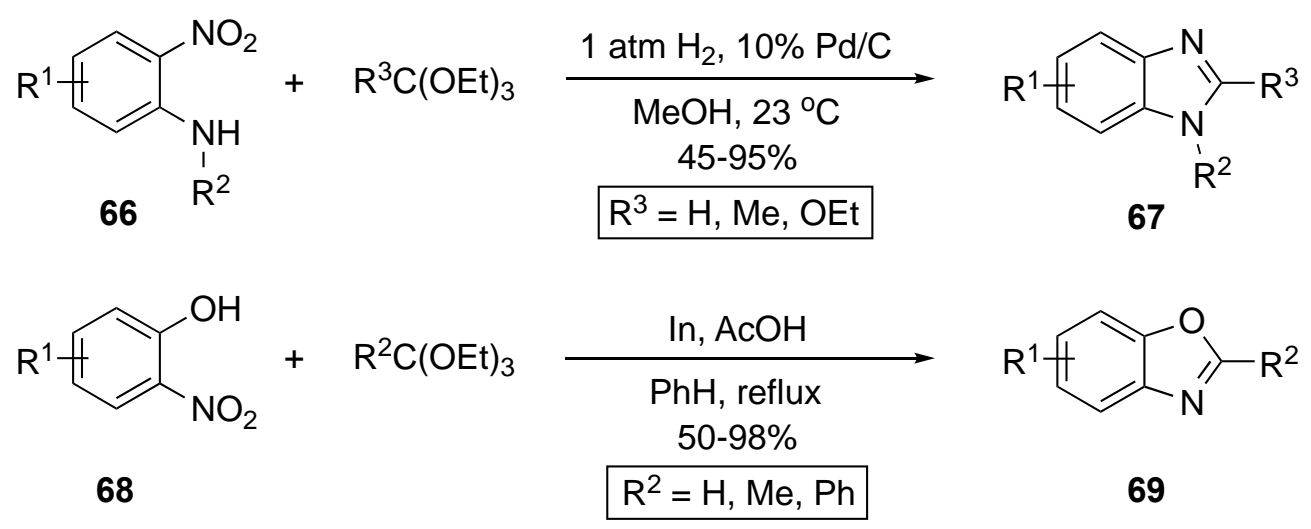

Scheme 18. Domino syntheses of benzimidazoles and benzoxazoles initiated by reduction of nitro precursors.

Chorvat and colleagues prepared and studied a series of 2,6,8,9-tetrasubstituted purines as corticotropin-releasing factor binding agents in an effort to enhance immune response to various types of stress. $^{33} \mathrm{~S}_{N} \mathrm{Ar}$ conversion of the symmetrical dichloroaminopyrimidine $\mathbf{7 0}$ to the arylamino-substituted derivative $\mathbf{7 1}$, followed by heating with a series of orthoesters, afforded imidazopyrimidine $\mathbf{7 2}$. Intermediate 72 was then reacted with a series of amines to produce the target compounds 73 (Scheme 19). Several of these purines exhibited good pharmaceutical properties.<smiles>[R7]c1nc2c(Cl)nc(C)nc2n1[Z17]</smiles>

Scheme 19. Synthesis of 2,6,8,9-tetrasubstituted purines.

A team led by Smith adapted the synthesis of similarly substituted purines to a solid-phase protocol. ${ }^{34}$ Starting with commercial ArgoGel-MB-CHO, several primary amines were loaded onto the gel by reductive amination. $S_{N} A r$ reaction of resin-bound secondary amine $\mathbf{7 4}$ with 4,6-dichloro-2-(methylthio)-5nitropyrimidine (75) linked the aromatic heterocycle to the resin to give 76. Similar replacement of the C-6 chlorine by an alkylamino moiety was followed by oxidation of the C-2 thioether to a sulfone. A third $S_{N} A r$ process served to install the final alkylamino unit at C-2 via displacement of the sulfone. Reduction of the nitro function using chromium(II) chloride in DMF/methanol (MeOH) gave 77 and heterocyclization with a triethyl orthoester then led to the resin-bound purine derivative $\mathbf{7 8}$. The target compound $\mathbf{7 9}$ was released from the resin bead by treatment with aqueous TFA at $23^{\circ} \mathrm{C}$. The purines were cleaved with good to excellent yields and purities, but the cyclization step was sensitive to steric hindrance when $R^{2}$ and $R^{4}$ were large. The reaction sequence for the assembly of purines $\mathbf{7 9}$ by this strategy, as well as number schemes for reactants and products, are summarized in Scheme 20. 


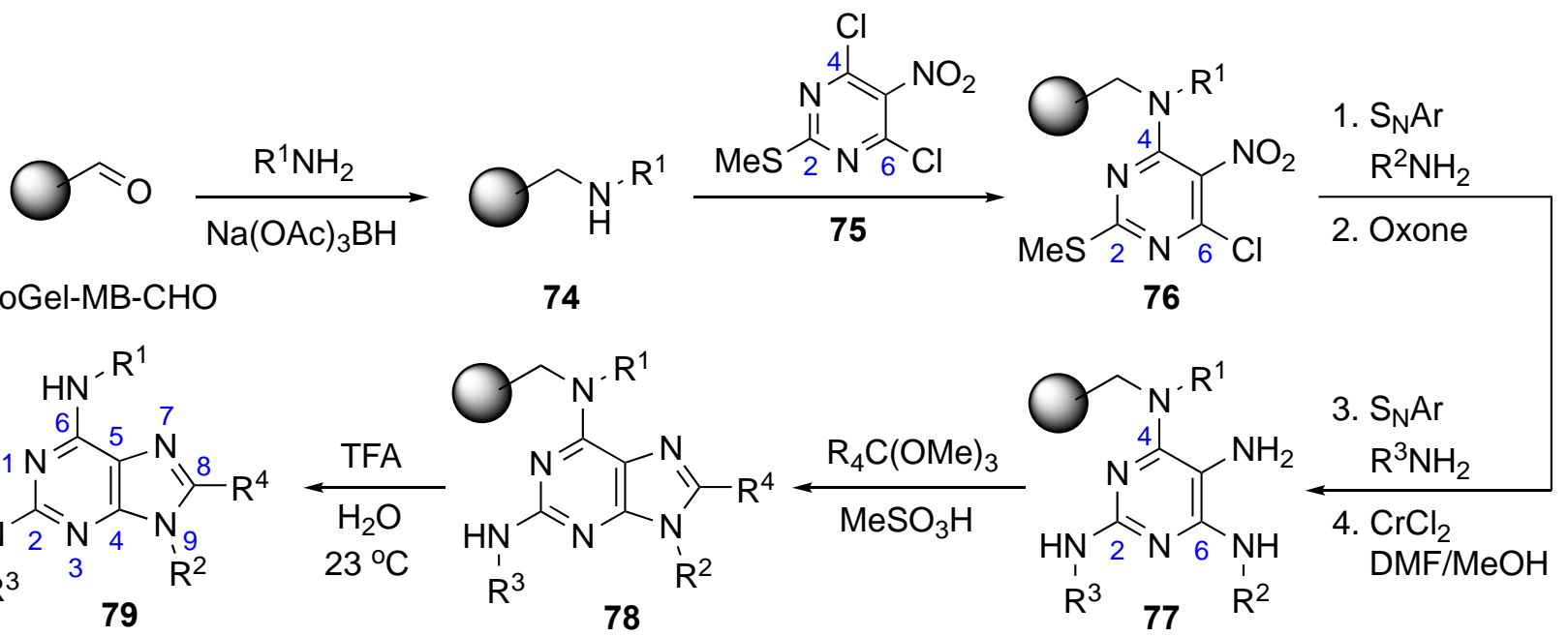

Scheme 20. Solid-phase protocol for the synthesis of tetrasubstitued purines.

Finally, a further example of this ring forming strategy was reported by Portilla et al. ${ }^{35}$ who applied MW heating to promote an annulation reaction to form pyrazolo[5,1- $b]$ purines. Using a novel scheme, several pyrazolo[1,5-a]pyrimidine-7-amines were prepared as cyclization precursors. Irradiation of 5-amino- $1 \mathrm{H}$ pyrazoles 80 with 2-arylhydrazinyliden-3-oxobutanenitriles 81 with $\mathrm{MW}$ [300 W, $\left.180{ }^{\circ} \mathrm{C}, 4 \mathrm{~min}\right]$ afforded the azo-substituted pyrazolo[1,5-a]pyrimidines 82 (>75\% yield). Catalytic reduction of the azo function [1 atm $\mathrm{H}_{2}$, $10 \% \mathrm{Pd} / \mathrm{C}, \mathrm{EtOH}, 60{ }^{\circ} \mathrm{C}, 24 \mathrm{~h}$ ] supplied the target diamines 83 in yields surpassing $90 \%$. Heterocyclization of 83 with triethyl orthoformate or orthoacetate [MW, $300 \mathrm{~W}, 110-120{ }^{\circ} \mathrm{C}, 5-10 \mathrm{~min}$ ] then provided the pyrazolo[5,1-b]purines 84 (5 cases) in $81-96 \%$ yields. The process is summarized in Scheme 21.

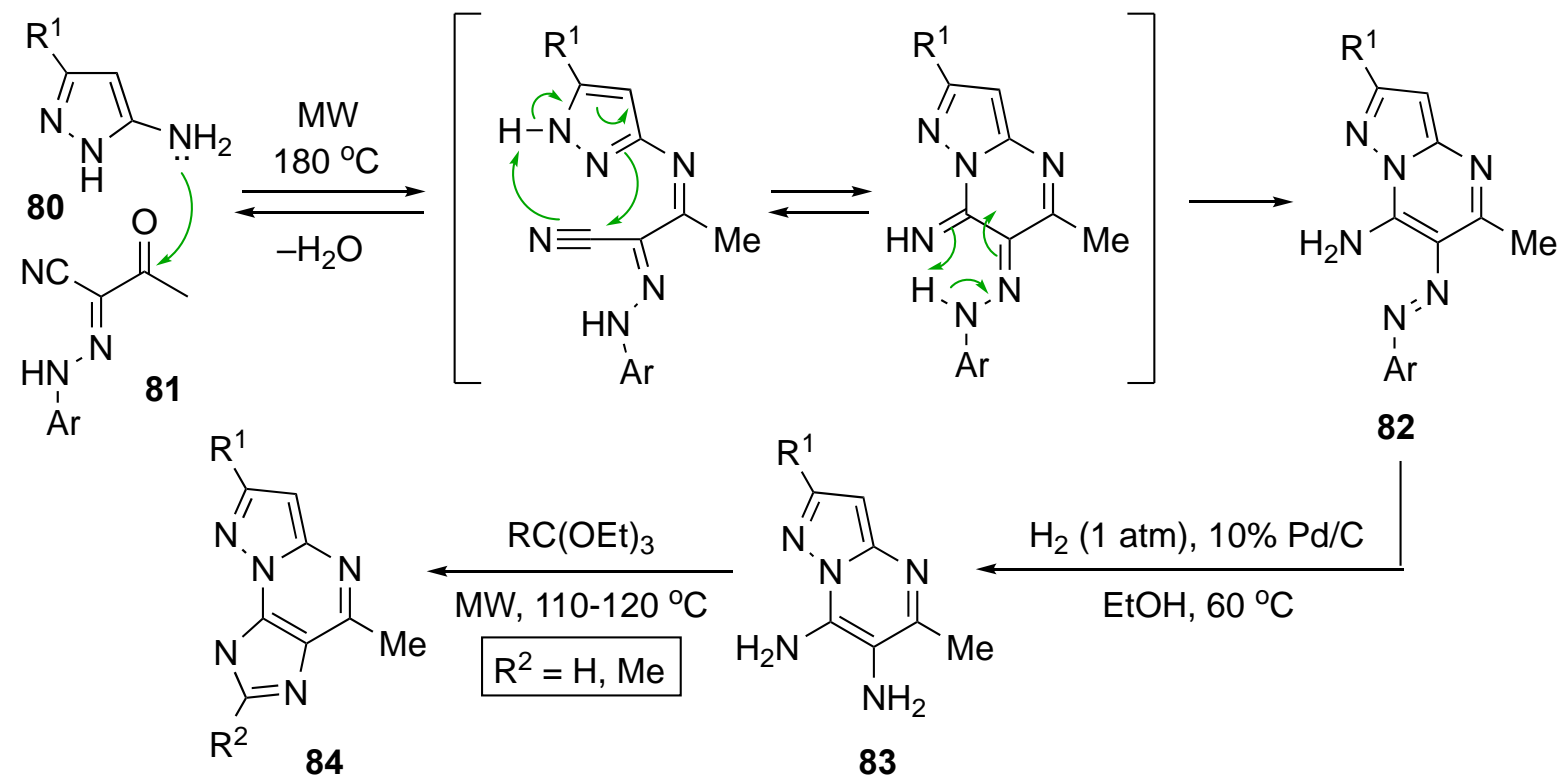

Scheme 21. Synthesis of pyrazolo[5,1-b]purines.

\subsection{Fused rings: three heteroatoms}

Numerous other methods for fusing five-membered rings with multiple heteroatoms to pre-existing rings have also been documented. Most of these have sought to generate compounds of medicinal value. A paper by 
McQuaid and co-workers ${ }^{36}$ utilized an orthoester for heterocycle annulation to a quinoxaline (Scheme 22). In a search for excitory amino acid antagonists which could be used to treat neurodegenerative diseases, 2hydrazinoquinoxaline 85 , prepared in 3 steps (44\%) from 4,5-dichloro-o-phenylenediamine, ${ }^{37}$ was cyclized with triethyl orthoformate to give the heteroannulated quinoxaline 86 in near quantitative yield. ${ }^{38}$ Final treatment of $\mathbf{8 6}$ with hydrogen peroxide in acetic acid (no yield given) produced 7,8-dichloro-1,2,4triazolo[4,3-a]quinoxalin-4(5H)-one (87), which proved to be one of the more potent drug candidates in their screening study.

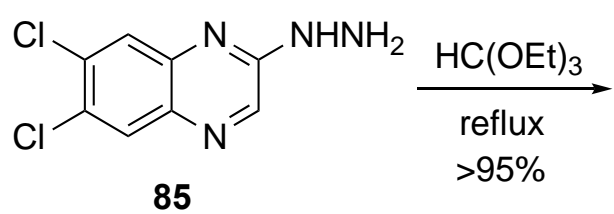

85<smiles>Clc1cc2ncc3nncn3c2cc1Cl</smiles>

86

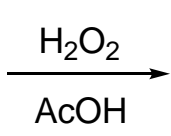<smiles>O=c1[nH]c2cc(Cl)c(Cl)cc2n2cnnc12</smiles>

87

Scheme 22. Synthesis of 7,8-dichloro-1,2,4-triazolo[4,3-a]quinoxalin-4(5H)-one (87).

A similar ring system was explored by Magnani et al. ${ }^{39}$ seeking drug candidates for the same neurodegenerative afflictions (Scheme 23). This procedure utilized precursor $\mathbf{8 8}$ wherein the lactam unit was already present, and a hydrazone moiety replaced the hydrazino group. Not only were these substrates more difficult to prepare ( 6 steps, $1.7 \%$ from 1 -indanone), but the yields of the cyclization products 89 were also much lower (21-29\%).

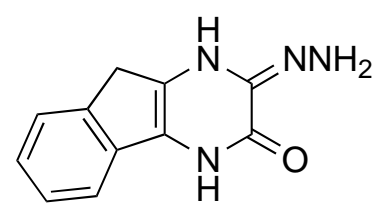

88

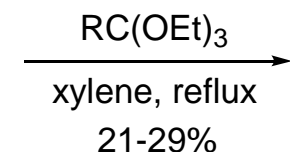

$\mathrm{R}=\mathrm{H}, \mathrm{Me}, \mathrm{Et}, \mathrm{Bu}$

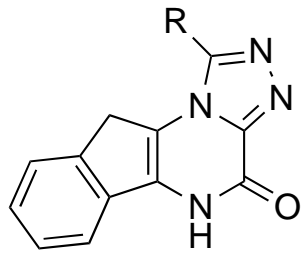

89

Scheme 23. Synthesis of $5 H, 10 H$-indeno[1,2-e]-1,2,4-triazolo[4,3-a]pyrazin-4-ones.

Liu and co-workers ${ }^{40}$ published an application of this transformation to prepare compounds that inhibit human platelet aggregation. The cyclization substrate, 2-hydrazinonaphthothiazine (90), was prepared in 4 steps (50\%) from 8-amino-1-naphthalenesulfonic acid. Heating $\mathbf{9 0}$ with a series of orthoesters at reflux for 30 min delivered the desired naphtho[1,8-de][1,2,4]triazolo[3,4-b][1,3]thiazines 91 in 72-82\% yield (Scheme 24). Although all of the derivatives showed inhibitory activity, only the compound derived from triethyl orthoformate was discussed in detail.

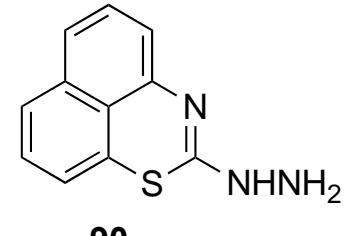

90

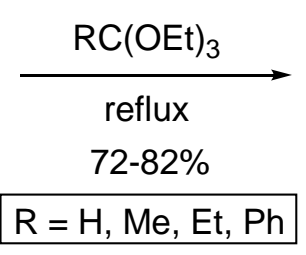

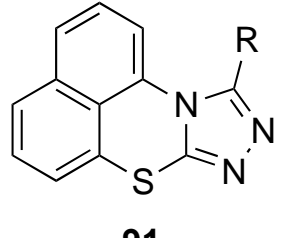

91

Scheme 24. Synthesis of naphtho[1,8-de][1,2,4]triazolo[3,4-b][1,3]thiazines. 
The Davoodnia group published a preparation of 1,2,4-triazolo[4,3-a]perimidine, the tetraaza analog of 91, but no further studies were described for this compound. ${ }^{41}$

Other related examples have appeared in the literature ${ }^{42-48}$ and are summarized in Figure 1. All reactions were performed neat or as solutions in the indicated solvent and at the reported temperature (room temperature was assumed to be $23^{\circ} \mathrm{C}$ ). The subunit derived from the orthoester is highlighted in red for each molecule.

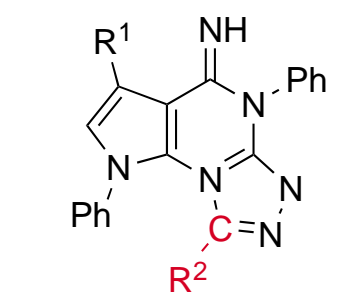

$92\left(\mathrm{R}^{2}=\mathrm{H}, \mathrm{Me}, \mathrm{Et}\right)$ neat, cat $\mathrm{AcOH}$, reflux, 3 h, 9 cases, 63-83\%

Ref. 42

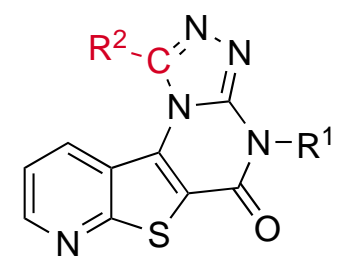

$95\left(R^{2}=H, M e, E t\right)$

$\mathrm{EtOH}$, cat $\mathrm{AcOH}$, reflux

$7 \mathrm{~h}, 5$ cases, 69-83\%

Ref. 46<smiles>[R]c1cn(-c2ccccc2)c2nc([R])n3c([R])nnc3c12</smiles>

$93\left(\mathrm{R}^{2}=\mathrm{H}, \mathrm{Me}, \mathrm{Et}\right)$ $\mathrm{EtOH}$, reflux $6 \mathrm{~h}$, 12 cases, $63-80 \%$

Refs. 43,44<smiles>[R7]c1nnc2n1nc(C)c1nc(C3CCCCC3)nn12</smiles>

$96\left(\mathrm{R}^{1}=\mathrm{H}, \mathrm{Me}\right)$ $\mathrm{EtOH}, 23^{\circ} \mathrm{C}, 8 \mathrm{~h}$ 5 cases, 51-67\%

Ref. 47<smiles>[R7]Nc1nc2c(s1)=NC1=NN=C([R7])C1C=2C</smiles>

$$
94\left(\mathrm{R}^{2}=\mathrm{H}, \mathrm{Me}\right)
$$

$\mathrm{MeCN}$, cat $\mathrm{AcOH}$, reflux, 2-4 h, 8 cases, 62-89\%

Ref. 45<smiles>[R]c1nnc2n1N=C([Al+2])CS2</smiles>

$97\left(\mathrm{R}^{1}=\mathrm{H}, \mathrm{Me}\right)$ TFA, $23^{\circ} \mathrm{C}, 12 \mathrm{~h}$, 9 cases, $61-96 \%$ Ref. 48

Figure 1. Other examples of fused 5-membered rings with three heteroatoms.

\subsection{Complex rings and systems that rearrange}

Many reactions that form five-membered rings undergo a rearrangement following the initial cyclization process. This bond reorganization is known as the Dimroth rearrangement, ${ }^{49}$ and several versions of the process have been cataloged. The process is detailed in Scheme 25 for 1,2,4-triazolo[4,3-a]pyridine (98) under basic conditions. ${ }^{49,50}$ At moderate temperatures $\left(100-120{ }^{\circ} \mathrm{C}\right)$, cyclization of 2-hydrazinopyridine with an orthoester initially gave 98 as the kinetic product, and this slowly rearranged to the more stable thermodynamic $[1,5-a]$ product 100 . When the reaction was repeated at higher temperature $\left(\geq 150{ }^{\circ} \mathrm{C}\right), 100$ was isolated as the major product. Under basic conditions, a ring bond in $\mathbf{9 8}$ is cleaved to generate the stabilized cyclic anion 99, which undergoes bond rotation and reclosure to the 1,2,4-triazolo[1,5-a]pyrimidine $100 .^{51}$ This process, however, is also observed in acid and in neutral solvents with heat where an anionic intermediate is unlikely. 


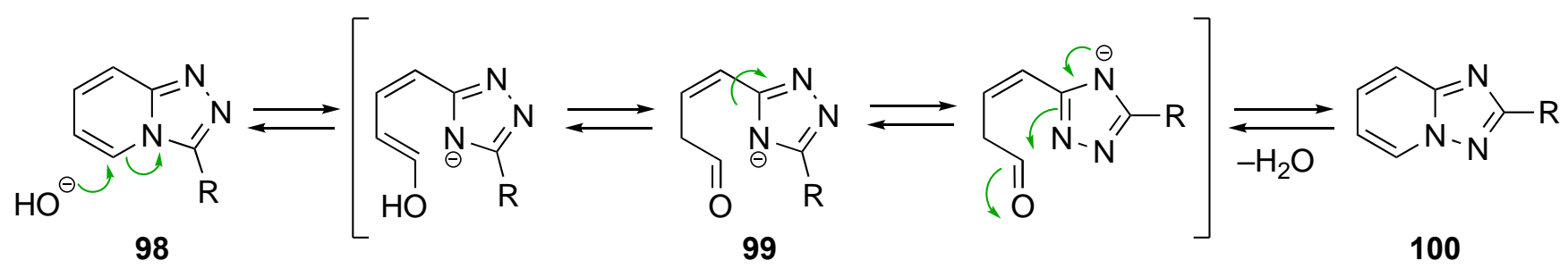

Scheme 25. Dimroth rearrangement of $\mathbf{9 8}$ to give $\mathbf{1 0 0 .}$

This same rearrangement was also noted in 1,2,4-triazolo[4,3-a]pyrimidines ${ }^{52}$ and 8-hydroxy-1,2,4triazolo[4,3-c]pyrimidines ${ }^{53}$ as well as in more complex ring systems, some of which are detailed below.

Brown and Shinozuka ${ }^{54}$ reported a synthesis of $s$-triazolo[4,3-c]pteridines and noted that most underwent a Dimroth rearrangement to their [1,5-c] isomers (Scheme 26). Treatment of pteridin-4ylhydrazine (101) with a selection of triethyl orthoesters in dioxane at reflux for 10-60 min yielded the 3-alkyls-triazolo[4,3-c]pteridines 102 in $>90 \%$ yield. Continued heating under the same conditions eventuated a Dimroth rearrangement to the 2-alkyl-s-triazolo[1,5-c]pteridines 103 in 16-95\% yield, with the lowest yields resulting from orthoesters having larger carbon-bound alkyl groups, e.g. orthoformate (95\%) > orthoacetate $(57 \%)>$ orthopropionate $(16 \%)$. In this example, no acid or base was present to facilitate the isomerization reaction. Pteridines 102 with $R=$ Me gave better conversions to the [1,5-c] isomers than those with $R=H$, but several, with substitution on the pteridine ring, required in excess of $40 \mathrm{~h}$ of heating.<smiles>NNc1ncnc2nccnc12</smiles>

101

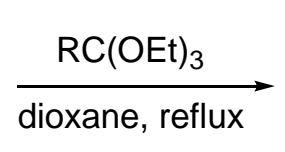<smiles>Fc1nnc2c3nccnc3ncn12</smiles>

102

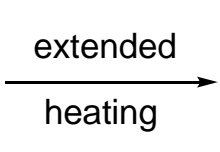

heating<smiles>Cc1nc2c3nccnc3ncn2n1</smiles>

103

Scheme 26. Synthesis of 2-alkyl-s-triazolo[1,5-c]pteridines.

Nagamatsu and Fujita ${ }^{55}$ observed a similar rearrangement in the conversion of 4-hydrazinylpyrimidin$2(1 H)$-one $(104)$ to 1,2,4-triazolo[4,3-c]pyrimidin-5(6H)-ones 105 . These heterocyclizations gave the [4,3-c] structures 105 in $64-81 \%$ yields when treated with the orthoester in TFA at $23-60{ }^{\circ} \mathrm{C}$ for $0.5-24 \mathrm{~h}$. Unfortunately, while the products were stable for several days in acidic media (TFA or conc. $\mathrm{HCl}$ ), it proved impossible to cleanly recrystallize these solids due to facile conversion to the [1,5-c] isomers 106 in warm solvents, such as EtOH or dimethyl sulfoxide (DMSO) (Scheme 27). A rate study was performed by ${ }^{1} \mathrm{H}-\mathrm{NMR}$ in DMSO- $d_{6}$, which revealed that for this ring system, larger $\mathrm{R}$ substituents accelerated the process (e.g. the rate for $\mathrm{R}=\mathrm{Ph}>\mathrm{Me}>\mathrm{H}$ ).<smiles>NNc1cc[nH]c(=O)n1</smiles>

104

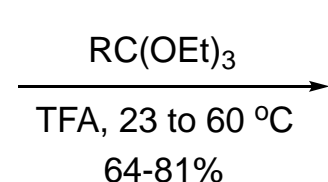

$64-81 \%$<smiles>[R]c1nnc2cc[nH]c(=O)n12</smiles>

105

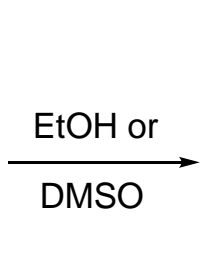<smiles>[R]c1nc2cc[nH]c(=O)n2n1</smiles>

106

Scheme 27. Synthesis and rearrangement of 1,2,4-triazolo[4,3-c]pyrimidin-5(6H)-ones. 
The Liu group ${ }^{56}$ took advantage of this cyclization-rearrangement sequence in a study to prepare a small set of potential antihypertensive agents. Several derivatives were surveyed, and the reaction is illustrated for the most active compound (Scheme 28). Treatment of 2-hydrazino-3-phenylquinazolin-4(3H)one (107) with triethyl orthoacetate in pyridine at reflux for $16 \mathrm{~h}$ afforded 2-methyl-4(phenylamino)[1,2,4]triazolo[2,3-a]quinazolin-5(4H)-one (109) in 39\% yield. This arose from an initial condensation to give the 1-methyl [4,3-a] product 108, followed by a Dimroth rearrangement to the [2,3-a] isomer. Compound 109 was observed to lower blood pressure by ca. $40 \%$ at a dose of $2 \mathrm{mg} / \mathrm{kg}$ in rats.

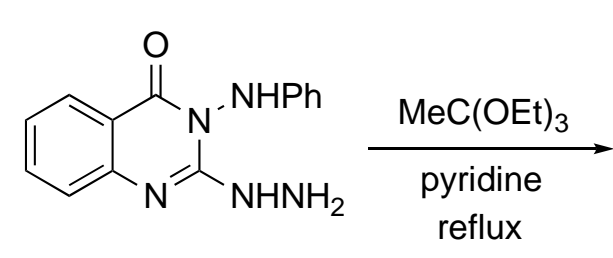

107<smiles>Cc1nnc2n(Nc3ccccc3)c(=O)c3ccccc3n12</smiles>

108

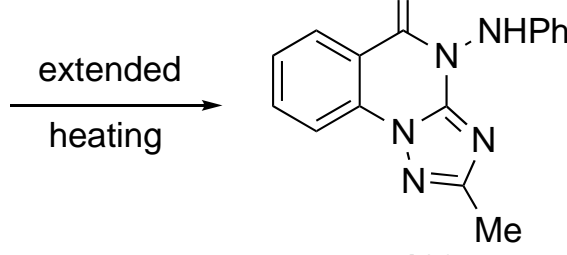

109

Scheme 28. Synthesis of 2-methyl-4-(phenylamino)[1,2,4]triazolo[2,3-a]quinazolin-5(4H)-one.

\section{Six-Membered Rings}

\subsection{Monocyclic/fused rings: two heteroatoms}

Two publications have appeared describing a synthesis of tri- and tetrasubstituted pyrimidines using orthesters (Scheme 29). ${ }^{57,58}$ In this work, 3-methyl-5-(trimethylsilyl)methylisoxazole (110) was treated with $n$ butyllithium and an aryl nitrile at $-70{ }^{\circ} \mathrm{C}$, followed after $1 \mathrm{~h}$ by a second aryl nitrile at $23{ }^{\circ} \mathrm{C}(30-90 \%)$. Cyclization of the resulting enamidines $\mathbf{1 1 1}$ with triethyl orthoformate or orthoacetate in the presence of catalytic zinc bromide in refluxing toluene (14-96 h) afforded the substituted pyrimidine derivatives 112 in 56$99 \%$ yield.

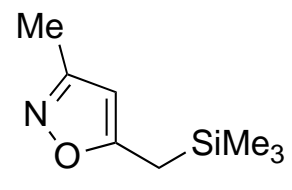

110

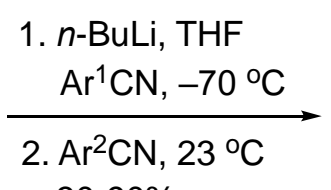

$30-90 \%$

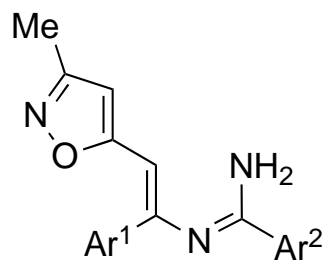

111

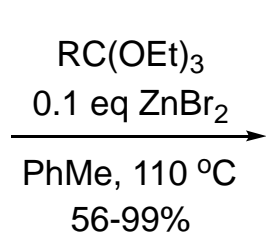

$\mathrm{R}=\mathrm{H}, \mathrm{Me}$

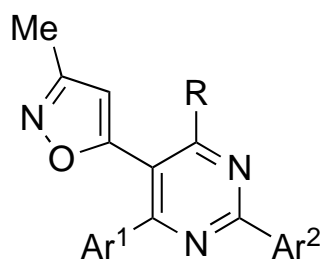

112

Scheme 29. Synthesis of tri- and tetrasubstituted pyrimidines.

The generation of fused rings from orthoesters has been directed primarily towards the formation of quinoxalines and benzoxazines. These are both highly coveted templates found in many drug molecules. The process to assemble these structures from 2-(aminomethyl)- or 2-(hydroxymethyl)anilines is usually quite facile since both reactive centers are held in close proximity by the aromatic ring. In 2-(aminomethyl)aniline, the two nitrogens have sufficient nucleophilicity to rapidly react with the orthoester as it sequentially loses alcohol molecules. In 2-(hydroxymethyl)aniline, the analogous cyclization is expected to be more difficult due the decreased nucleophilicity of oxygen and the shorter $\mathrm{C}-\mathrm{O}$ bond length. Additionally, in products with a 
carbonyl adjacent to the oxygen, as in $4 H$-benzo[d][1,3]-benzoxazin-4-ones, the greater reactivity of the ester versus the amide linkage would make the heterocycle less stable.

One of the earliest disclosures of a cyclization involving insertion of an orthoester between two nitrogens to form a six-membered ring was advanced in 1960 by Partridge and co-workers (Scheme 30). ${ }^{59}$ These investigators found that refluxing 2-(2-aminophenyl)-4-hydroxyquinoline (113a) with triethyl orthoformate for $2 \mathrm{~h}$ led to clean formation of $114 \mathrm{a}$ (86\%). The authors asserted that triethyl orthoformate promoted a more efficient ring closure than an earlier cyclization with formic acid, ${ }^{60}$ but no yield was recorded in the cited work. A similar conversion was documented by these same researchers from the corresponding 4(phenylamino) derivative $\mathbf{1 1 3 b}$ to generate the 4 -(phenylimino) product $114 \mathrm{~b}$ in $76 \%$ yield. $^{61}$
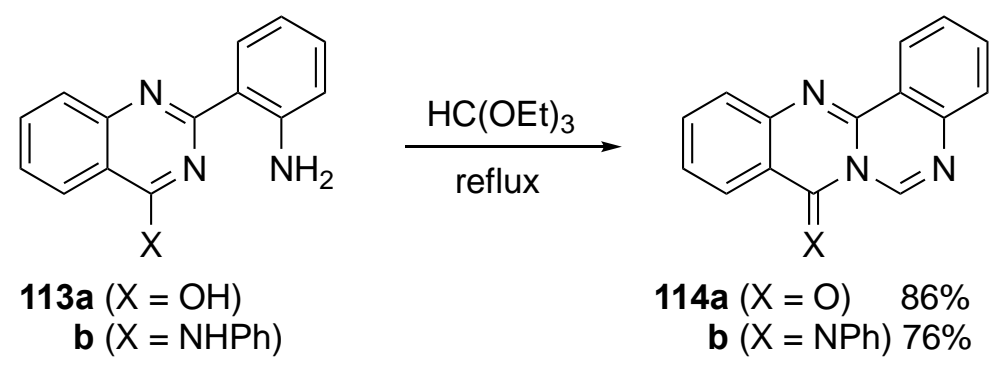

Scheme 30. Synthesis of tetracycles 114a and 114b.

The Bunce group has published an improved procedure for the preparation of quinazolin-4(3H)-ones. ${ }^{62}$ This method examined cyclizations of 2-aminobenzamide (115) with a series of orthoesters promoted by $\mathrm{AcOH}$. Best results were observed when the reaction was performed in $\mathrm{EtOH}$ with $\mathrm{AcOH}\left(2-3\right.$ equiv) at $110{ }^{\circ} \mathrm{C}$ in a sealed tube. Under these conditions, steric factors seemed to pose less of a problem, and very high yields were achieved with minimal purification requirements. Although the study was focused on procedure rather than scope, the reaction was found to be tolerant of a small selection of substituents on the aromatic ring and the amide nitrogen of the substrate. The ring formation is illustrated with a mechanism in Scheme 31 and follows a path parallel to that described for the formation of benzimidazoles. ${ }^{24}$ Initial loss of EtOH from the orthoester and attack by the aromatic amino function of $\mathbf{1 1 5}$ on the stabilized carbonium ion would give 116. Protonation of 116, followed by loss of a second molecule of EtOH, should give 117, which can cyclize to 118. Elimination of a third molecule of EtOH would then furnish the quinazolin-4(3H)-one 119. This account also described an extension of this process to the preparation of 5,6-dihydropyrimidin-4(3H)-ones, but the yields were modest for all examples except when $\mathrm{R}$ was $\mathrm{Ph} .^{62}$

El-Gaby et al. utilized orthoesters in a novel approach to pyrimidothienopyridazines. ${ }^{63}$ Synthesis of the cyclization substrate 5-amino-3-methyl-4-styryl-6-carbamoylthienyl[2,3-c]pyridazine (120) was achieved in five steps (57\%) from 5,6-dimethyl-3-oxo-2,3-dihydropyridazine-4-carbonitrile. ${ }^{64}$ This precursor was boiled in triethyl orthoformate containing catalytic AcOH for $1 \mathrm{~h}$ to afford 3-methyl-4-styryl-7,8-dihydro-8oxopyrimido[4',5':4,5]thieno[2,3-c]pyridazine (121) in 96\% yield (Scheme 32). Two other derivatives bearing aryl substitution on the amide nitrogen were also prepared, each in $90 \%$ yield. 


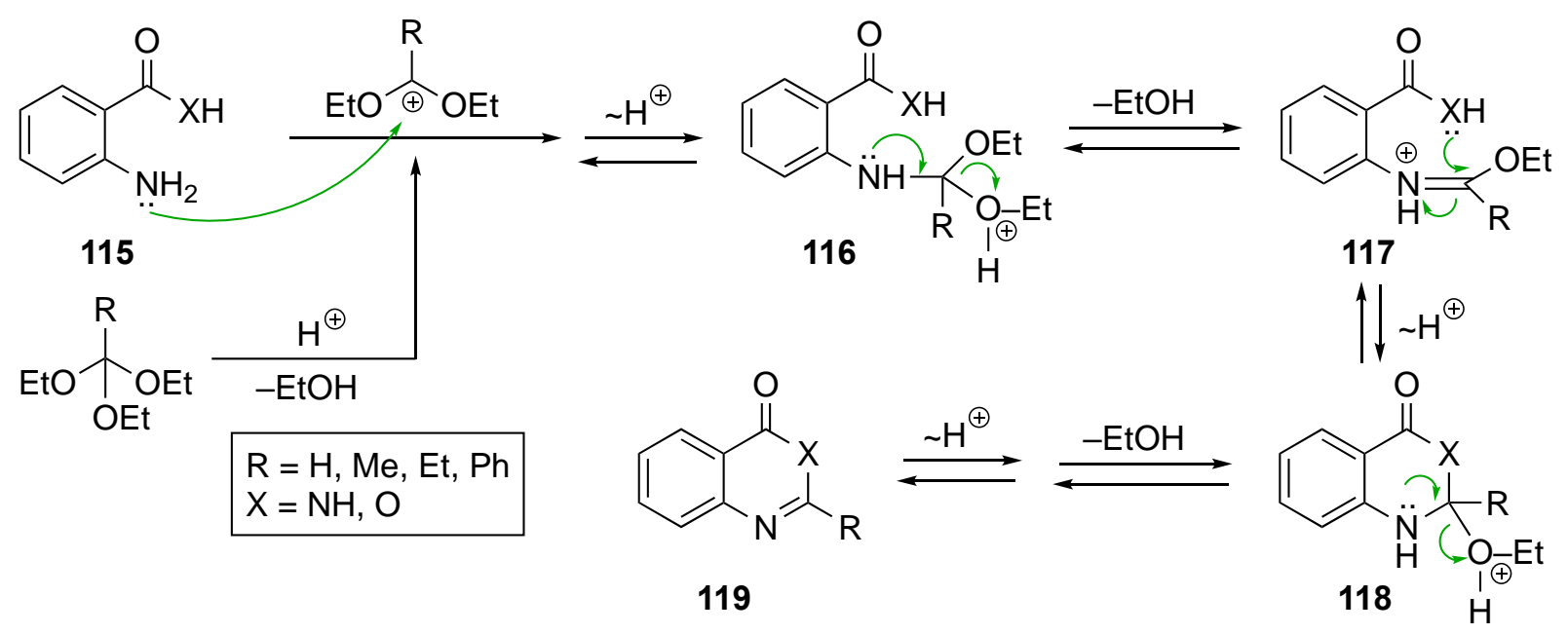

Scheme 31. Synthesis of quinazolin-4(3H)-ones.<smiles>Cc1nnc2sc(C(N)=O)c(N)c2c1/C=C/c1ccccc1</smiles>

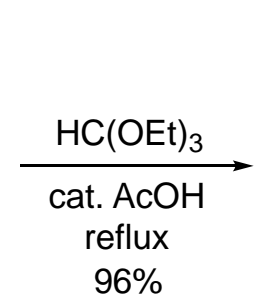<smiles>Cc1nnc2sc3c(=O)[nH]cnc3c2c1/C=C/c1ccccc1</smiles>

Scheme 32. Synthesis of 3-methyl-4-styryl-7,8-dihydro-8-oxopyrimido[4',5':4,5]thieno[2,3-c]pyridazine.

Two research groups have also documented several other 6-ring cyclizations from orthoesters (Figure 2). In the first case, Hazarkhani and Karimi $^{65}$ cyclized a series of orthoesters with 2-amino- $N$ benzimidazolylbenzamide in DMA using catalytic $p$-toluenesulfonic acid ( $p$-TsOH) under MW conditions to produce 3-(2-benzimidazolyl)-4(3H)-quinazolinones 122 in 70-95\% yields. Davoodnia ${ }^{66}$ exploited MW heating to promote the cyclization of 2-(1H-benzimidazol-2-yl)aniline to the benzimidazo[1,2-c]quinazolines 123 in 82 $88 \%$ yields. Experimental and spectral details in this report were minimal. This same group ${ }^{67}$ further examined the ring closure of thieno[2,3-d]pyrimidin-4(3H)-ones 124 (73-82\%) promoted by phosphotungstic acid. The catalyst was shown to be recyclable, with a slight decrease in yield for a single subsequent cyclization. Davoodnia and co-workers also reported the synthesis of pyrazolo[3,4- $d$ ]pyrimidin-4-ones 125 from 5-amino1-phenyl-1H-pyrazole-4-carboxamides and orthoesters without solvent using catalytic Brønsted acid ionic liquids (84-93\%) ${ }^{68}$ and montmorillonite $\mathrm{K} 10(78-89 \%){ }^{69}$ Again, the catalysts were touted as being reusable, but it was not clear how many cycles could be performed with the same charge of catalyst. Additionally, a tedious extraction procedure was necessary to recycle the ionic liquid catalyst. The final products are depicted below, once again showing the fragment derived from the orthoester in red. 


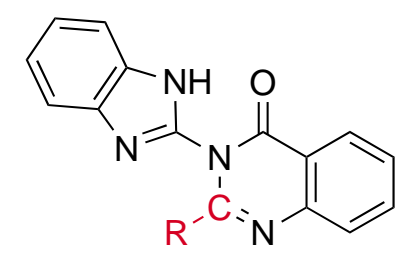

$122(\mathrm{R}=\mathrm{H}, \mathrm{Me}, \mathrm{Et}, \mathrm{Bu})$ $\mathrm{MeC}(\mathrm{O}) \mathrm{NMe}_{2}, p-\mathrm{TsOH}$, MW $600 \mathrm{~W}, 30 \mathrm{~s}$, 4 cases, $70-95 \%$ yields

Ref. 65<smiles>[R]c1nc2sc([R])c([R])c2c(=O)[nH]1</smiles>

$124\left(\mathrm{R}^{3}=\mathrm{H}, \mathrm{Me}, \mathrm{Et}\right)$

$\mathrm{EtOH}, \mathrm{H}_{3}\left[\left(\mathrm{PW}_{3} \mathrm{O}_{10}\right)\right]_{4}, 20-$

30 min, 6 cases, $73-82 \%$

Ref. 67<smiles>[R]c1nc2ccccc2c2nc3ccccc3n12</smiles>

$123(\mathrm{R}=\mathrm{H}, \mathrm{Me}, \mathrm{Et})$ neat, MW $800 \mathrm{~W}, 60-80$ min, 3 cases, $82-88 \%$

Ref. 66<smiles>[R]c1nc2c(cnn2[Ga])c(=O)[nH]1</smiles>

$125(\mathrm{R}=\mathrm{H}, \mathrm{Me}, \mathrm{Et})$ neat, Brønsted acid ionic liquids, $80^{\circ} \mathrm{C}, 2-30 \mathrm{~min}, 7$ cases, $84-93 \%$ or neat montmorillonite $\mathrm{K} 10,100^{\circ} \mathrm{C}$,

7-25 min, 6 cases, $78-89 \%$ Refs. 68,69

Figure 2. Other 6-membered rings formed using orthoesters.

On rare occasions, unexpected reactions are observed in cyclizations with orthoesters. One such example was noted by Okamoto, et al. in the ring closure of 2-alkoxy-5-(benzimidazol-2-ylidene)-3-cyano-6imino-5,6-dihydropyridines 126 with triethyl orthoesters at reflux to produce 6-alkyl-3-alkoxy-2-cyano4,5,6a,11-tetraazabenzo[a]fluorene derivatives 127 (Scheme 33). ${ }^{70}$ While 127 was obtained cleanly from reaction with triethyl orthoformate, cyclizations from the orthoacetate and orthopropionate were complicated by the unexpected transfer of an ethyl group from an orthoester oxygen to C-1 of the heterocycle to give 128 upon prolonged heating. Furthermore, it was observed that product 127 from triethyl orthoformate was ethylated when boiled with triethyl orthoacetate. The authors rationalized these observations in terms of the increased reactivity of the C-alkylated orthoesters and an initial ipso ethylation at $\mathrm{C}-2$, followed by migration of the ethyl substituent to $\mathrm{C}-1$.<smiles>[R7]C(OCC)c1nc(N)c(-c2nc3ccccc3[nH]2)cc1C#N</smiles><smiles>[R7]c1nc2nc(O)c(C#N)cc2c2nc3ccccc3n12</smiles>

127 (product when $\mathrm{R}^{2}=\mathrm{H}$ )

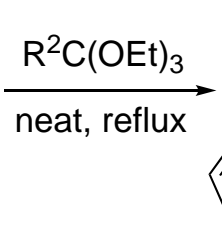<smiles>[R2]c1nc2nc([R7])n3c4ccccc4nc3c2c([14CH3])c1C#N</smiles>

(product when $\mathrm{R}^{2}=\mathrm{Me}, \mathrm{Et}$ )

Scheme 33. Synthesis of 4,5,6a,11-tetraazabenzo[a]fluorene derivatives 127 and 128. 
A paper by Shi and co-workers ${ }^{71}$ delineated a versatile domino reaction for the formation of quinazoline derivatives. In this procedure, a pre-formed low-valent titanium reagent, prepared from titanium(IV) chloride and zinc dust in THF, was treated with $\mathrm{N}$-aryl-2-nitrobenzylamines 129 or $\mathrm{N}$-aryl-2nitrobenzamides 131 and an orthoester. This process, performed in refluxing THF, involved the in situ reduction of the nitro function to the amino group, followed by condensation with the orthoester to give 3,4dihydroquinazolines 130 and quinazolin-4(3H)-ones 132, respectively (Scheme 34). These products were isolated in good to excellent yields.
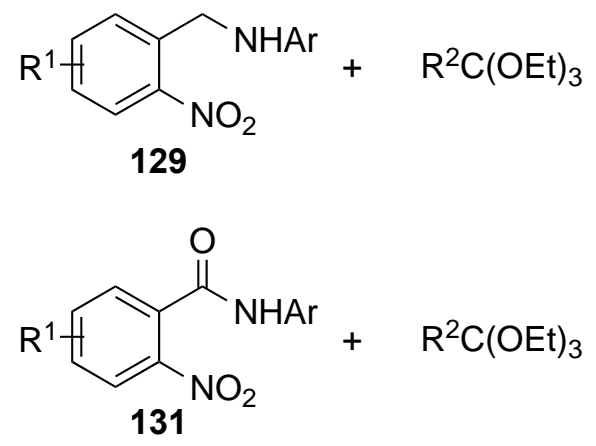

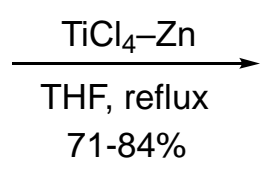

$\underset{\substack{\text { THF, reflux } \\ 71-86 \%}}{\stackrel{\mathrm{TiCl}_{4}-\mathrm{Zn}}{\longrightarrow}}$<smiles>[R]C1=Nc2cc[R1]([AsH3])cc2CN1</smiles>

130<smiles></smiles>

132

Scheme 34. Domino reduction-cyclization to form 3,4-dihydroquinazolines 130 and quinazolin-4(3H)-ones 132.

This same study ${ }^{71}$ extended this nitro reduction-cyclization strategy to the cyclization of 2-(2nitrophenyl)imidazoles 133 to imidazo[1,2-c] quinazolines 134, which proceeded in 61-74\% yields (Scheme 35). The procedure to prepare 133 was not described or referenced. However, the presence of a nitro group on the phenyl ring could potentially simplify the synthesis of many precursors since it would allow engagement of the $S_{N} A r$ reaction.

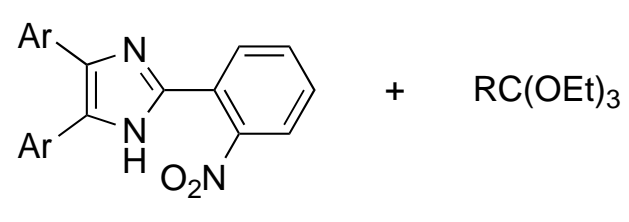

133

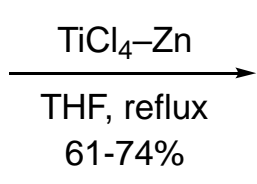

$61-74 \%$<smiles>[R]c1nc2ccccc2c2nc([Al])c([Al])n12</smiles>

134

Scheme 35. Domino reduction-cyclization to form imidazo[1,2-c]quinazolines.

An article by Agrebi et al. has appeared chronicling the use of an orthoester to generate a reactive intermediate that was converted to pyrazolo[3,4-d]pyrimidines. ${ }^{72}$ In this account, 5-aminopyrazole-4carbonitrile (135) was converted to its imidate 136 using triethyl orthoformate, orthoacetate or orthopropionate (25-70\%). Reaction of the imidate with ammonia in EtOH containing catalytic AcOH then provided 1-phenyl-1H-pyrazolo[3,4- $d$ ]pyrimidin-4-amines 137 (68-83\%). Aromatic amines in the second step provided the Dimroth rearranged products 138 in $37-84 \%$ yield (Scheme 36 ). 


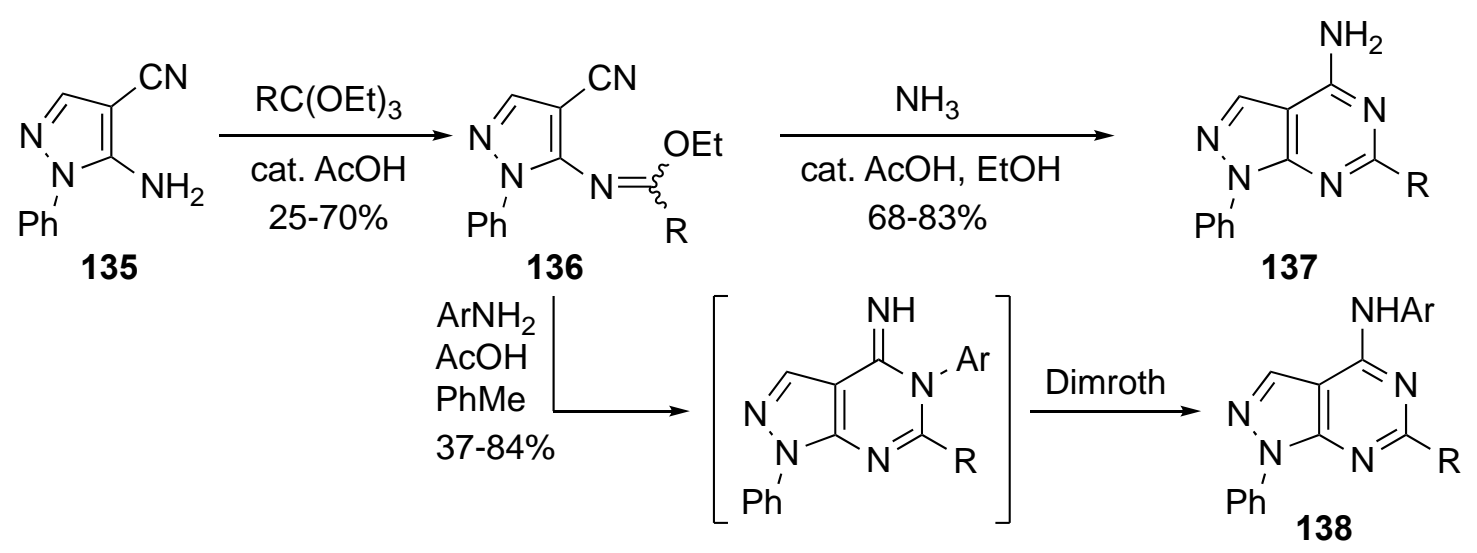

Scheme 36. Synthesis of pyrazolo[3,4-d]pyrimidines.

Davoodnia and co-workers have demonstrated the utility of orthoesters in the elaboration of 2-methyl5-aminoisoxazole-4-carboxamide (139) to isoxazolo[5,4-d]pyrimidin-4(5H)-ones 140 (Scheme 37)..$^{73}$ Two substrates were reacted with triethyl orthoformate and orthoacetate under neat conditions with finely ground silica sulfuric acid catalyst. With conventional heating, yields were good (63-67\%), but reaction times were $\geq 120 \mathrm{~min}$. Under microwave heating (700 W, no temperature given), comparable yields (66-82\%) were achieved, but with dramatically shorter reaction times ( $\leq 10 \mathrm{~min})$. These authors also reported the preparation isoxazolo[5,4-d][1,3] oxazines from 5-amino-3-methylisoxazole-4-carboxylic acid. ${ }^{74}$<smiles>Cc1noc(N)c1C(N)=O</smiles>

139

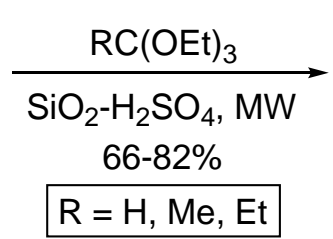<smiles>[R]c1nc2onc(C)c2c(=O)[nH]1</smiles>

140

Scheme 37. Synthesis of isoxazolo[5,4-d]pyrimidin-4(5H)-ones.

Annor-Gyamfi and Bunce ${ }^{75}$ recently disclosed the application of this reaction to aromatic amino acids. In this study, several anthranilic acids $\mathbf{1 4 1}$ were reacted with orthoesters (2.7 equiv) under neat conditions in the presence of stoichiometric $\mathrm{AcOH}$ at $100{ }^{\circ} \mathrm{C}(4-48 \mathrm{~h})$ to generate $4 \mathrm{H}$-benzo[d][1,3] oxazin-4-ones 142 . Interestingly, not all of the substrates explored afforded the desired targets. It was noted that anthranilic acids with electron-rich aromatic rings produced $4 \mathrm{H}$-benzo[d][1,3] oxazin-4-ones, while those bearing electronwithdrawing substituents tended to stop at the dihydro derivatives 143, wherein the final elimination of EtOH did not occur. Reaction of 2-aminonicotinic acid also produced the dihydro product. The reaction was further explored using microwave conditions $\left[400 \mathrm{~W}, 100{ }^{\circ} \mathrm{C}\right.$ ] with nearly identical results, although reaction times were significantly shorter (0.75-3 h) (Scheme 38). The formation of the dihydro derivative was linked to the availability of the electron pair on nitrogen. In electron-deficient aromatic rings, these electrons would be less available to assist in the elimination of the final molecule of EtOH. In several substrates, it was possible to isolate either product by modification of the reaction conditions. Similar dihydro derivatives were previously observed in a synthesis of highly substituted 2,3,4,5-tetrahydro-1,2,4-triazines from 2arylhydrazonoacetamides and the same rationale can be applied. ${ }^{76}$ 


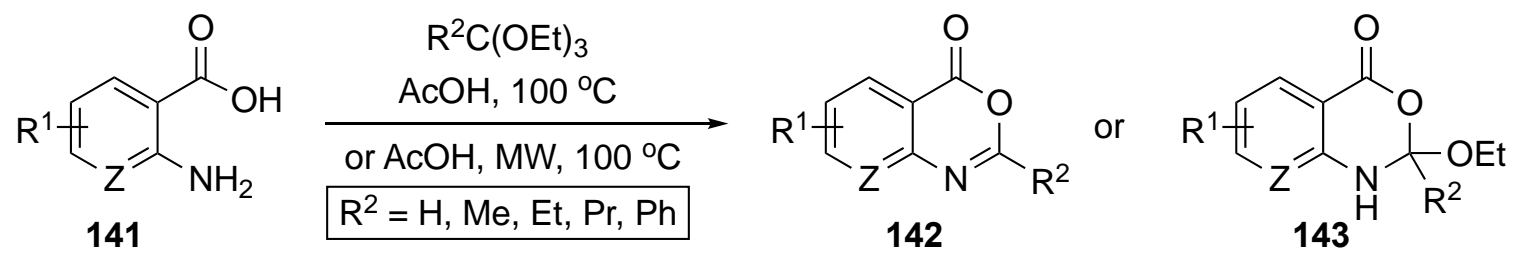

Scheme 38. Synthesis of $4 H$-benzo[d][1,3]oxazin-4-ones.

In 1973, Fujii and co-workers ${ }^{77}$ communicated an early orthoester-based approach to an adenine derivative (Scheme 39). In this work, imidazole $144^{78}$ was cyclized with boiling triethyl orthoformate to produce 145 in 73\% yield. ${ }^{77}$ Hydrogenolysis of the N-methoxy group from the perchlorate salt of $145\left[\mathrm{H}_{2}, 10 \%\right.$ $\mathrm{Pd} / \mathrm{C}$ ] then afforded 3,9-dimethyladenine perchlorate in $26 \%$ yield.<smiles>C/N=C(\N)c1c(NC)ncn1C</smiles>

144

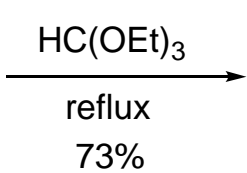

$73 \%$

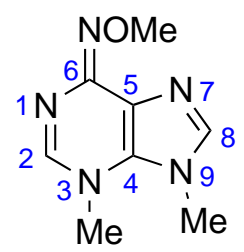

145

Scheme 39. Synthesis of an adenine derivative.

\subsection{Monocyclic/fused: three heteroatoms}

In the late 1980 's, Piskala ${ }^{79}$ led a project to obtain 5-azacytosines as potential antisecretory agents for the mitigation of ulcers. The preparation involved the treatment of amidinourea hydrochloride (146) with ethyl orthobenzoate in DMF at $100{ }^{\circ} \mathrm{C}$ for $1.5 \mathrm{~h}$ to give a $52 \%$ conversion to the drug candidate 147 (Scheme 40). As this was part of a patent, few details were available.

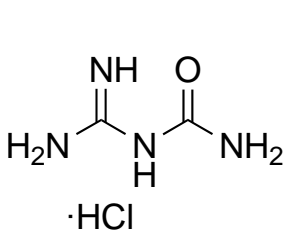

146

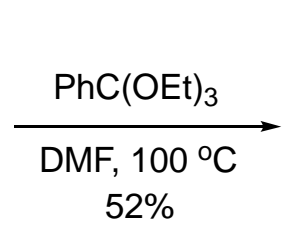

$52 \%$

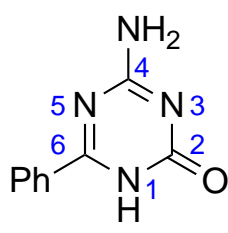

147

Scheme 40. Synthesis of a 5-azacytosine drug candidate.

An investigation by Zemanová and co-workers ${ }^{80}$ sought to access furo-fused pyrrolo[1,2$d][1,2,4]$ triazine derivatives as potential antibacterials. Starting from methyl $4 H$-furo[3,2-b]pyrrole-5carboxylate (148), hydrazinolysis afforded the hydrazide 149 in high yield. ${ }^{81}$ Treatment of 149 with triethyl orthoformate (1 equiv) in dry DMF for $3.5 \mathrm{~h}$ afforded furo[2',3':4,5]pyrrolo[1,2-d][1,2,4]triazin-8(7H)-one (150) in 69\% (Scheme 41). Among the compounds evaluated, 150 expressed the highest activity versus a panel of Gram-positive and Gram-negative bacteria and was significantly better than the 6-aminopenicillanic acid used as a standard. The compound derived from triethyl orthoacetate was also prepared (71\%), but was less active. 
<smiles>COC(=O)c1cc2occc2[nH]1</smiles>

148

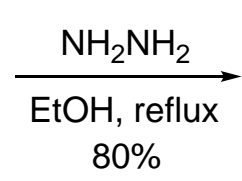

$80 \%$<smiles>NNC(=O)c1cc2occc2[nH]1</smiles>

149

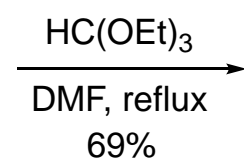

$69 \%$

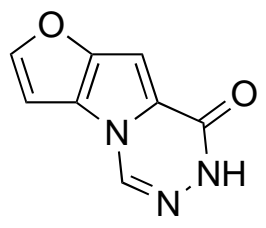

150

Scheme 41. Synthesis of furo-fused pyrrolo[1,2-d] [1,2,4]triazine derivatives as potential antibacterials.

An additional example of a fused ring with three heteroatoms was generated from chiral alcohol $151 .{ }^{82}$ Coogan and co-workers treated alcohol $\mathbf{1 5 1}$ with a series of orthoesters to furnish optically pure $4 \mathrm{H}-3-\mathrm{oxa}-$ 1,9a,10-triazaanthracen-9-ones 152 in 54-77\% yields (Scheme 42). ${ }^{83}$ At the time, these products constituted the first members of this previously unknown heterocyclic ring system.
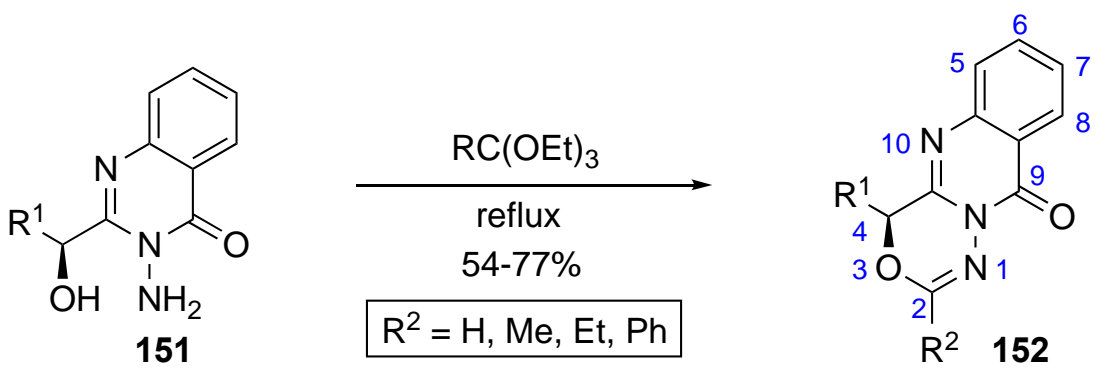

Scheme 42. Synthesis of optically pure 4H-3-oxa-1,9a,10-triazaanthracen-9-ones.

In another study, 1,3-bisnucleophile 154, prepared from phenyl cyanomethanesulfonate (153), was reacted with $\beta$-aminovinyl methyl ketone (155) followed by ammonia [150 ${ }^{\circ} \mathrm{C}$, pressure vessel, $48 \mathrm{~h}$ ] to give phenyl 2-amino-6-methylpyridine-3-sulfonamide (156) in very poor yield (4\%). Subsequent condensation of 156 with triethyl orthoformate and orthoacetate at $45^{\circ} \mathrm{C}$ for $2 \mathrm{~h}$ afforded 6-methyl- and 3,6-dimethyl-4Hpyrido[2,3-e][1,2,4]thiadiazine-1,1-dioxides (157a) and (157b), respectively, in $38 \%$ and $87 \%$ yields (Scheme 43). ${ }^{84}$ These targets were sought as selective potassium channel openers and were aza-isosteres of a known antihypertensive agent, but the overall yield was deemed insufficient for further development.

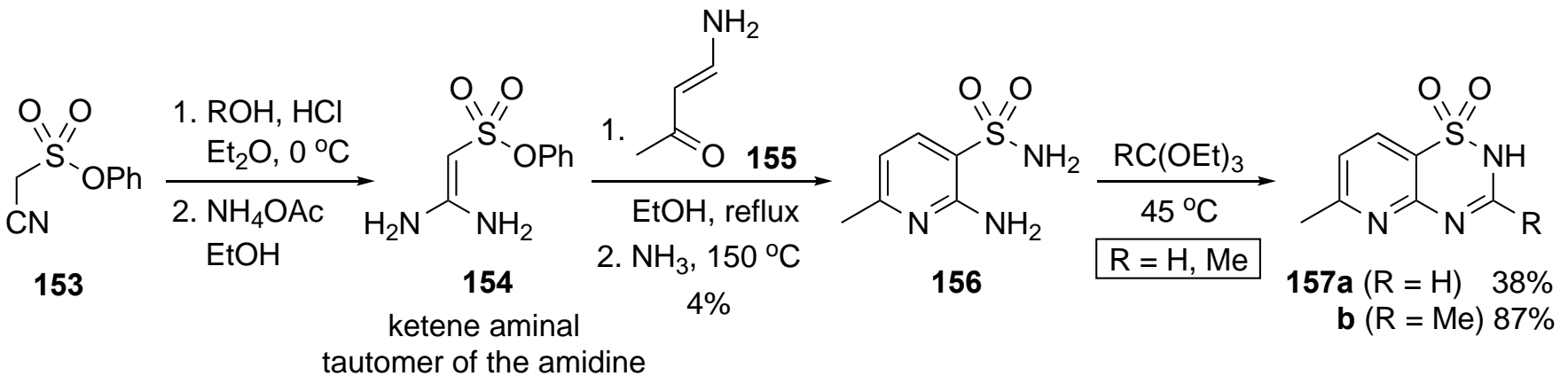

Scheme 43. Synthesis of $4 H$-pyrido[2,3-e][1,2,4]thiadiazine-1,1-dioxides.

\subsection{Monocyclic/fused: four heteroatoms}

Haake and Schümmelfeder offered a high-yield synthesis of a broad selection of thiatriazine rings (Scheme 44). ${ }^{85}$ Diphenyl $\mathrm{N}$-sulfamoylcarbonimidate (160) was supplied in $67 \%$ yield by condensation of 
dichlorodiphenoxymethane (158) with sulfamide (159) in acetonitrile at $0-20{ }^{\circ} \mathrm{C}$ for $48 \mathrm{~h}$. Reaction of 160 with bisnucleophiles such as ethylenediamine, 2-aminoethanol and 2-aminoethanethiol afforded imid-, ox- and thiazolidines $161 \mathrm{a}-\mathrm{c}$, respectively, in $77-89 \%$ yields. Reaction of $161 \mathrm{a}-\mathrm{c}$ furnished the bicyclic thiatriazines $162 a-c$ in $71-92 \%$ yields. In an alternative sequence, 160 was reacted with various primary and secondary amines, followed by ammonia, to give sulfamoylguanidines 163. Refluxing 163 with triethyl orthoformate or orthoacetate containing $p$-TsOH gave the 1,2,4,6-thiatriazine-1,1-dioxides 164 in 54-96\% yields. These compounds were evaluted as potential anti-ulcer drugs.

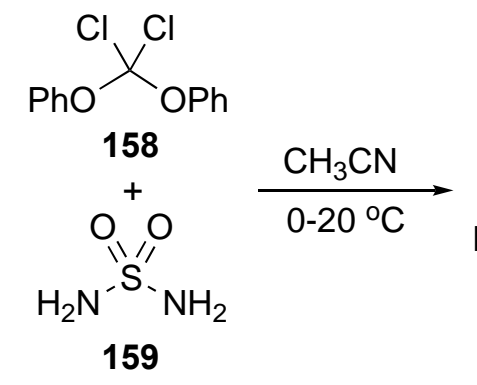

1) $\mathrm{R}^{1} \mathrm{R}^{2} \mathrm{NH}, i-\mathrm{PrOH}, 20^{\circ} \mathrm{C}$ 2) $\mathrm{NH}_{3}, i-\mathrm{PrOH}, 40^{\circ} \mathrm{C}$<smiles>NS(=O)(=O)N=C(Oc1ccccc1)Oc1ccccc1</smiles>

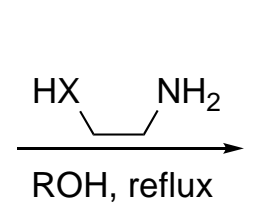<smiles>[X]C1=C(N)S(N)(=O)=C1NCC</smiles>

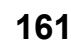

161a $(X=N H)$
b $(X=O)$ c $(X=S)$

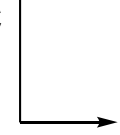

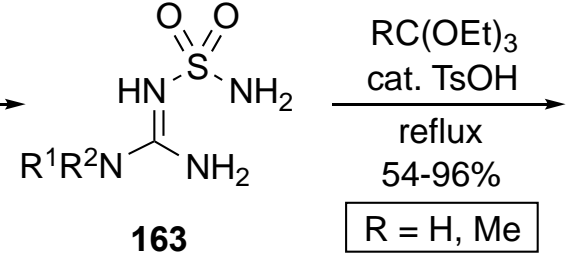
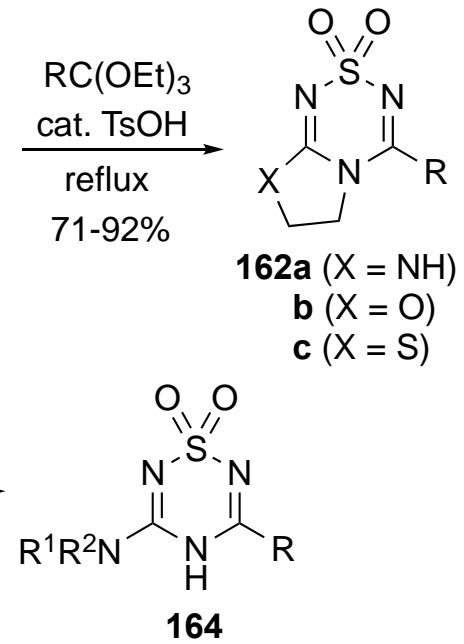

Scheme 44. Synthesis of a selection of thiatriazines.

Tetrazines often show biological activity, and thus, are attractive targets for heterocyclic and medicinal chemists. An approach to fused tetrazines has been described wherein 4-amino-6-methyl-3-hydrazino-4,5dihydro-1,2,4-triazine-5-thione (165) was prepared and reacted with triethyl orthoformate and orthoacetate to give the fused tetrazines 166 in $78 \%$ and $58 \%$ yields, respectively (Scheme 45 ). ${ }^{86}$<smiles>Cc1ncc(NN)n(N)c1=S</smiles>

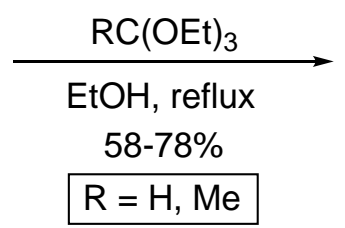<smiles>[R]C1=NNc2cnc(C)c(=S)n2N1</smiles>

Scheme 45. Orthoester approach to tetrazines.

\subsection{Complex Ring Systems}

A study by Sun and co-workers ${ }^{87}$ employed orthoesters in an electrocyclic ring closure-elimination sequence to obtain non-steroidal anti-inflammatory drug candidates (Scheme 46). Treatment of the 3aminobenzofuranylcoumarin 167 with triethyl orthoesters at reflux initially gave imino ether 168, followed by a $6 \pi$ electrocyclic ring closure and elimination of $\mathrm{EtOH}$ to give the fused coumarins 169 (70-80\%). Several of the products showed significant anti-inflammatory properties. 


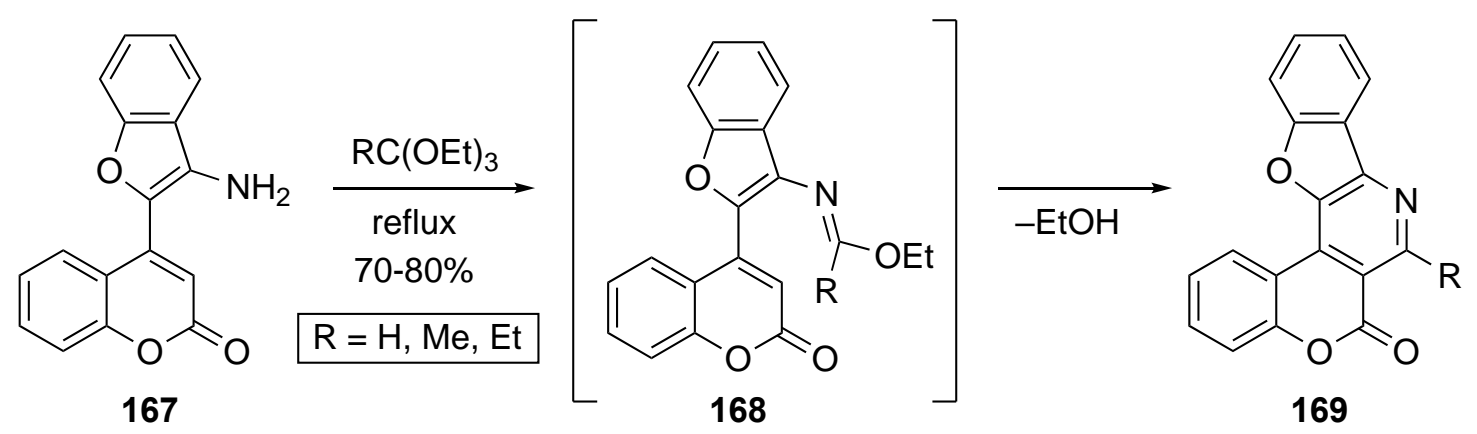

Scheme 46. Synthesis of non-steroidal anti-inflammatory drug candidates.

Additional reports have appeared ${ }^{88,89}$ describing the condensation of triethyl orthoacetate with $4 \mathrm{H}$ benzo[ $h$ ]chromene-3-carboxylates, such as $\mathbf{1 7 0}$, under reflux and microwave conditions to give the imino ether 171. Cyclization of $\mathbf{1 7 1}$ with arylhydrazines, under the same conditions, gave 9-anilino-10-methyl-7phenyl-7,9-dihydro-8H-benzo[7,8]chromeno[2,3-d]pyrimidin-8-ones $\mathbf{1 7 2}$ in 38\% overall yield (Scheme 47). Many cases were reported, with similar yields.<smiles>CCOC(=O)C1=C(N)Oc2c(ccc3ccccc23)C1c1ccccc1</smiles>

170<smiles>CCOC(=O)C1=C(/N=C(\C)OCC)Oc2c(ccc3ccccc23)C1c1ccccc1</smiles>

171<smiles>Cc1nc2c(c(=O)n1NCCl)C(c1ccccc1)c1ccc3ccccc3c1O2</smiles>

$2 \quad 172$

Scheme 47. Synthesis of 9-anilino-10-methyl-7-phenyl-7,9-dihydro-8H-benzo[7,8]chromeno[2,3- $d]$ pyrimidin-8ones.

Toche and co-workers ${ }^{90}$ described an interesting conversion that followed divergent paths through a common intermediate under base and acid conditions. A three-component reaction between 5 -amino- $1 \mathrm{H}$ pyrazole-4-carbonitrile (173), $p$-substituted benzoylacetonitriles 174 and triethyl orthoesters was performed in toluene containing catalytic triethylamine at reflux for $2 \mathrm{~h}$. The reaction proceeded through proposed intermediate 175, derived from an initially formed imino ether, to yield 7-arylpyrazolo[1,5-a]pyrimidine-3,6dicarbonitriles 176 (76-88\%). When the same reaction was carried out in EtOH containing catalytic $\mathrm{HCl}$, the process was hypothesized to traverse the same intermediate to deliver 7-amino-6-aroylpyrazolo[1,5a]pyrimidine-3-carbonitrile 177 (74-89\%). Thus, from intermediate 175, the triethylamine induced condensation of $\mathrm{N}-1$ of the pyrazole with the side chain carbonyl, while $\mathrm{HCl}$ favored $\mathrm{N}-1$ attack at the nitrile. Six examples of each cyclocondensation were reported and all gave the respective products cleanly in excellent yields (Scheme 48). 
<smiles>N#Cc1cn[nH]c1N</smiles>

173

$+$<smiles>O=C([Te])C[Al]</smiles>

174
$\mathrm{R}=\mathrm{H}, \mathrm{Me}, \mathrm{Et}$

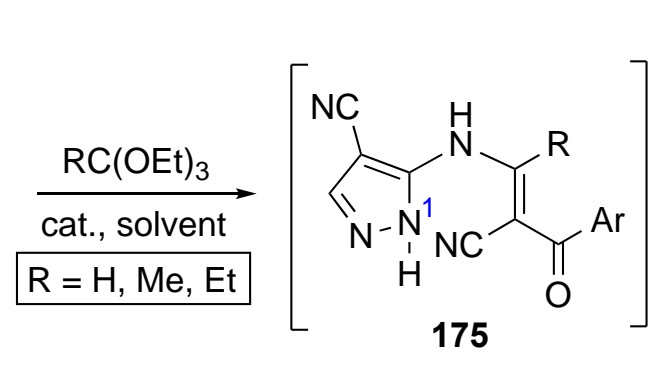

175

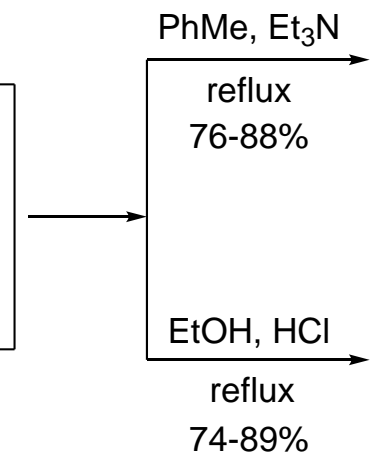

NC<smiles>[R]c1nc2c(C)cnn2c([Al])c1C#N</smiles>

176<smiles>[R]c1nc2c(C#N)cnn2c(N)c1C(=O)[Al]</smiles>

177

Scheme 48. Synthesis of $\mathbf{1 7 6}$ and $\mathbf{1 7 7}$ via divergent pathways through the same intermediate.

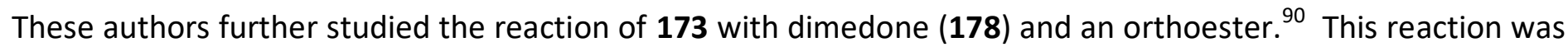
suggested to proceed via 179 to give 8,8-dimethyl-6-oxo-6,7,8,9-tetrahydropyrazolo[1,5-a]quinazoline-3carbonitriles $\mathbf{1 8 0}$. The yields of $\mathbf{1 8 0}$ were in the range of $82-89 \%$ (Scheme 49 ).<smiles>CC1(C)CC(=O)CC(=O)C1</smiles>

178

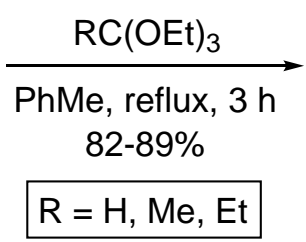<smiles>[R]C(Nc1c(C#N)cnn1C)=C1C(=O)CC(C)(C)CC1=O</smiles>

179

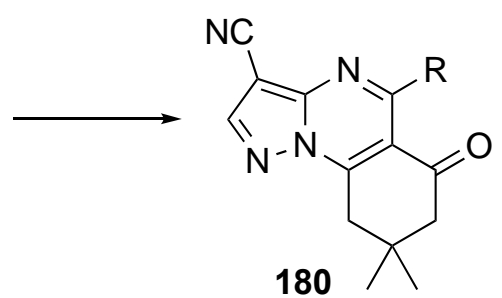

Scheme 49. Synthesis of 8,8-dimethyl-6-oxo-6,7,8,9-tetrahydropyrazolo[1,5-a]quinazoline-3-carbonitriles.

In a separate report, imino ethers 181, obtained from 2-amino-1-methyl-4,5-diphenyl-1H-pyrrole-3carbonitrile and triethyl orthoformate or orthoacetate, were cyclized with hydrazine to give 4-imino- $3 \mathrm{H}$ pyrrolo[2,3-d] pyrimidin-3-amines 182. Subsequently, 182 was cyclized with alkyl and aromatic acid chlorides in the presence of catalytic 4-(dimethylamino)pyridine (DMAP) to produce the pyrrolo[3,2e] $[1,2,4]$ triazolo[1,5-c]pyrimidines $183 .{ }^{91}$ The three-step procedure proceeded in $>73 \%$ for 9 examples (Scheme 50).

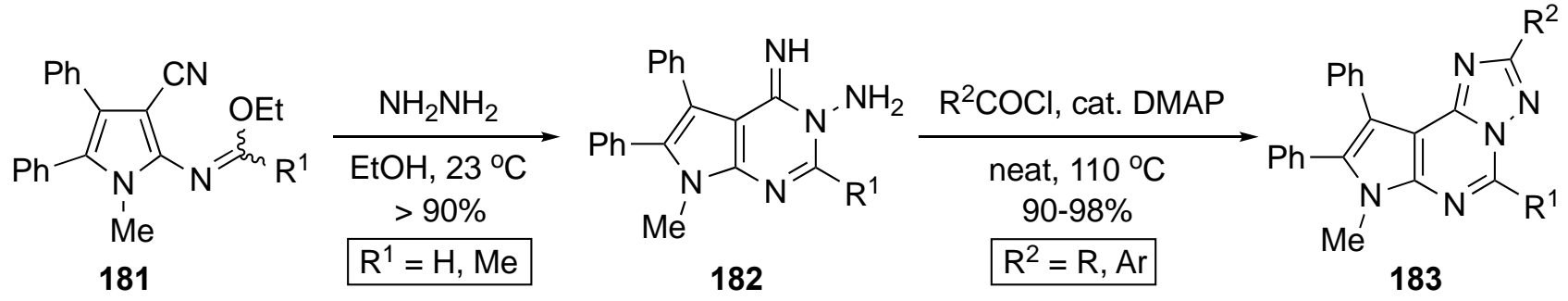

Scheme 50. Synthesis of pyrrolo[3,2-e][1,2,4]triazolo[1,5-c]pyrimidines. 
Two articles have described the use of orthoester condensations to form the purine six-membered ring. Initially, ${ }^{92}$ it was found that stirring formamidines 184 with catalytic 1,8-diazabicyclo[5.4.0]undec-7-ene (DBU) in EtOH resulted in $>90 \%$ conversion to imidazoles 185 . Further treatment of 185 with triethyl orthoformate closed the fused pyrimidine rings to give 9-aryl-6-cyano-9H-purines 186 in 69-91\% (Scheme 51).

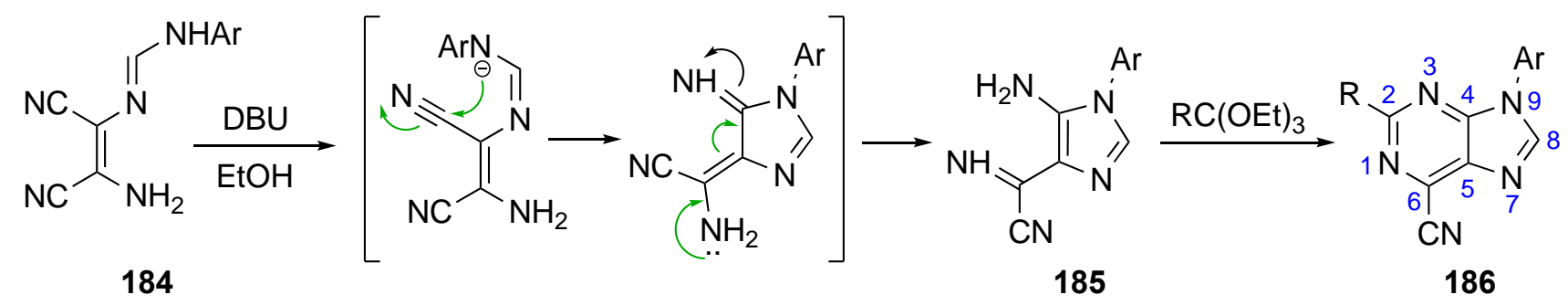

Scheme 51. Synthesis of 9-aryl-6-cyano-9H-purines.

Formation of the six-membered ring in this synthesis $(\mathbf{1 8 5} \rightarrow \mathbf{1 8 6})$ could also involve a $6 \pi$ electrocyclization via an imino ether intermediate 187 as shown in Scheme 52. Following ring closure, elimination of ethanol would deliver 186.

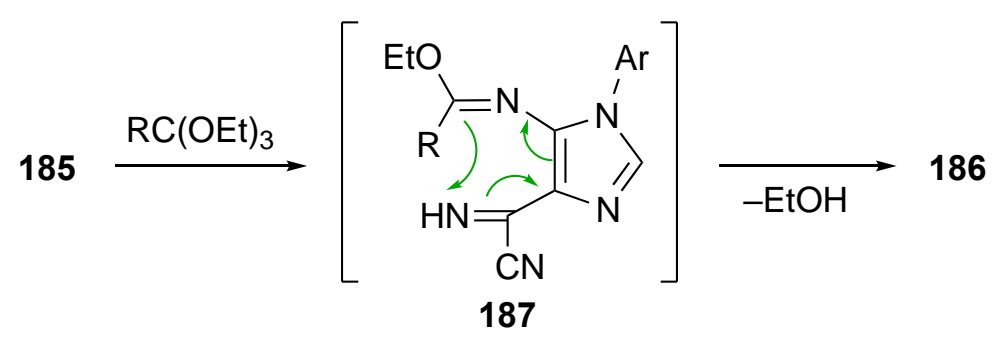

Scheme 52. Alternate pathway for generation of 186.

In a similar process, condensation of ethyl acetoacetate (188) with 185 in the presence of DBU afforded the 9-aryl-2-methyl-9H-purine-6-carboxamide $190 .^{93}$ Initial formation of 189 , followed by conversion to the amide by a previously described mechanism, ${ }^{94}$ would furnish product 190 (Scheme 53).

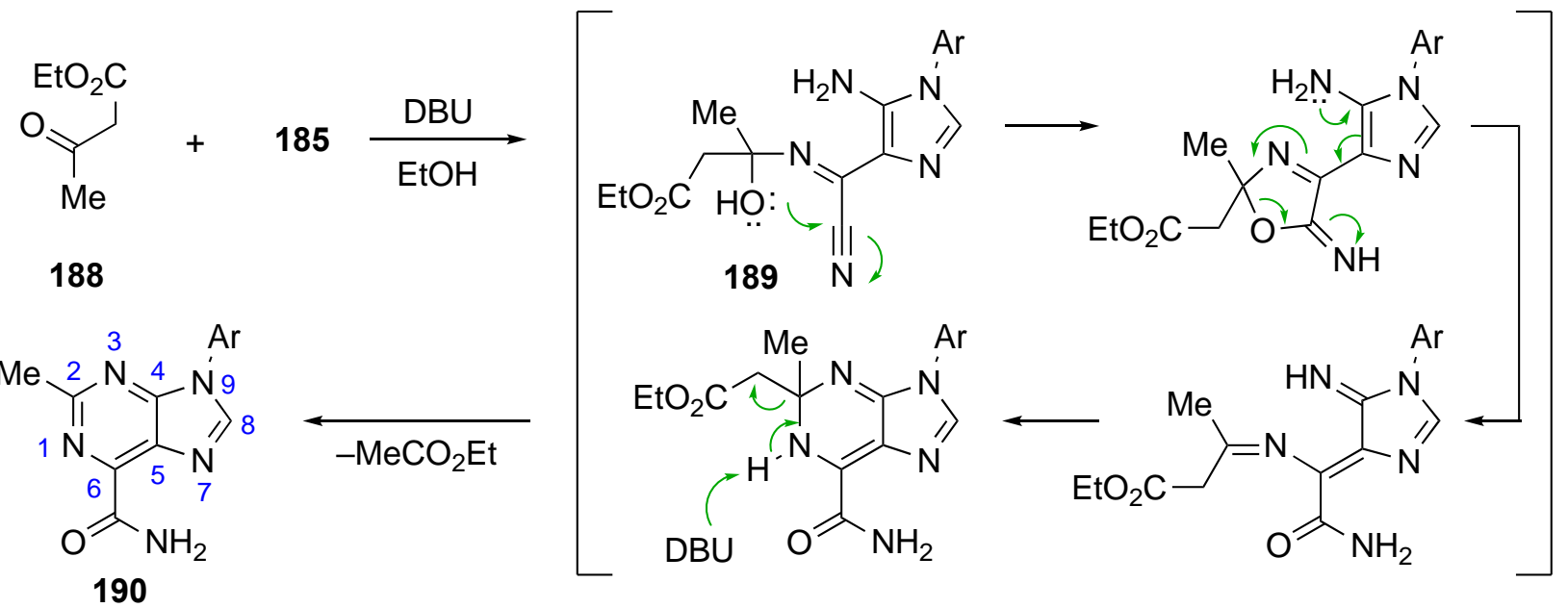

Scheme 53. Synthesis of 9-aryl-2-methyl-9H-purine-6-carboxamides 190. 
A related study ${ }^{95}$ revealed that reaction of formamidine 191 in refluxing triethyl orthoacetate or orthopropionate for $2 \mathrm{~h}$ resulted in the formation of two products, a 9-aryl-6-cyano-9H-purine 186 (25-43\%) and a 2-alkyl-1-aryl-1H-imidazole-4,5-dicarbonitrile 192 (51-66\%). The purine 186 arose from the process above for $184 \rightarrow \mathbf{1 8 6}$, while the imidazole resulted from condensation of the orthoester across the 1,3oriented amine nitrogens in 191 (Scheme 54). The $\mathrm{C}=\mathrm{N}$ in 191 is likely in tautomeric equilibrium with the conjugated form.<smiles>N#CC(N)=C(N)[Mg]</smiles>

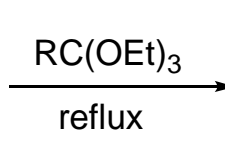

191<smiles>[R]c1nc(C#N)c(C#N)n1C=[W]</smiles>

192

Scheme 54. Alternative route to 9-aryl-6-cyano-9H-purines 186.

One last report detailed a complementary approach to prepare Tenofovir, an HIV/AIDs and hepatitis B drug. ${ }^{96}$ In this application, the imidazole ring of the purine was assembled first followed by annulation of the aromatic ring to give the adenine nucleus. Thus, reaction of diaminomaleonitrile (193) was condensed with trimethyl orthoformate in DMF containing catalytic TFA to give formidate 194 (93\%). Addition of $(R)$-1-aminopropan-2-ol to 194 in acetonitrile at $25{ }^{\circ} \mathrm{C}$ followed by treatment with barium hydroxide (1.2 equiv) at $-10{ }^{\circ} \mathrm{C}$ delivered key intermediate (R)-5-amino-1-(2-hydroxypropyl)- $1 \mathrm{H}$-imidazole-4-carbonitrile (HPI, 195) in $85 \%$ yield. Further condensation of 195 with formamidine acetate in DMF at $110{ }^{\circ} \mathrm{C}$ generated 196 (93\%). Finally, alkylation of 196 with ((tosyloxy)methyl)phosphoric acid in the presence of sodium tert-butoxide (7 equiv) at 5 ${ }^{\circ} \mathrm{C}$ with warming to $20{ }^{\circ} \mathrm{C}$ overnight afforded $(R)-(((1-(6-a m i n o-9 H$-purin-9-yl)propan-2-yl)oxy)methyl)phosphonic acid (Tenofovir, 197) in $70 \%$ yield (Scheme 55$)$.
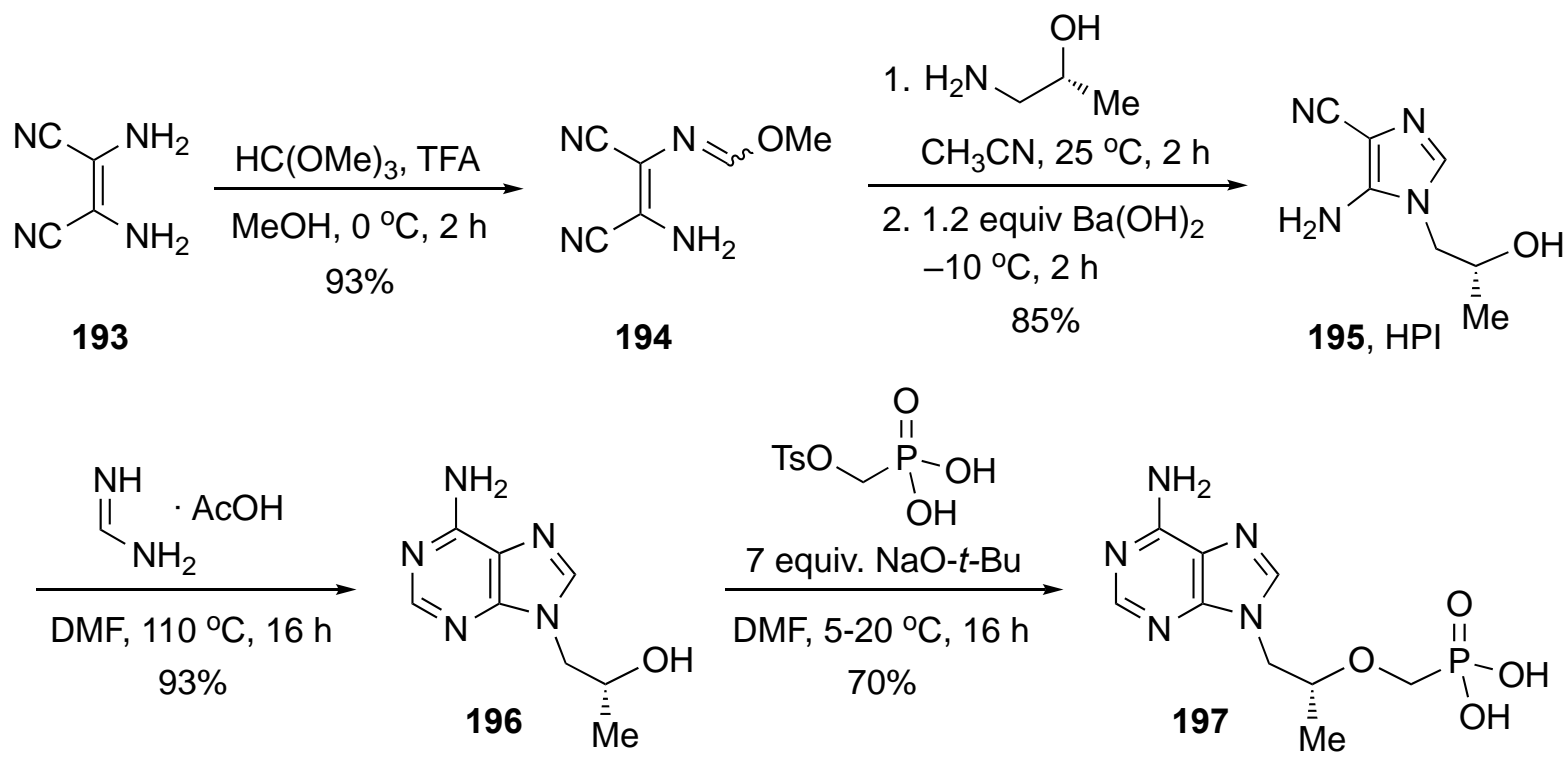

Scheme 55. Synthesis of HPI (195) and its conversion to the HIV/AIDS and hepatitis B drug Tenofovir (197). 


\section{Seven-Membered Rings}

The formation of seven-membered rings is rare and has only been reported one time. ${ }^{97}$ In this disclosure, Peet and co-workers initially reacted isatoic anhydride (198) with methylhydrazine in DMF to give 2-amino- $N$ methylbenzoic hydrazide (199). Subsequent treatment of 199 with a series of orthoesters in ethanol then led to the isolation of 3,4-dihydro-5H-1,3,4-benzotriazepin-5-ones 200a-d in $46-63 \%$ yields (Scheme 56). The success of this transformation was likely facilitated by the rigid planar structure which positioned the reactive centers in close proximity.<smiles>O=c1[nH]c2ccccc2c(=O)o1</smiles>

198

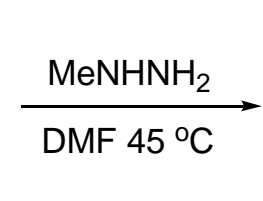

(19)

199

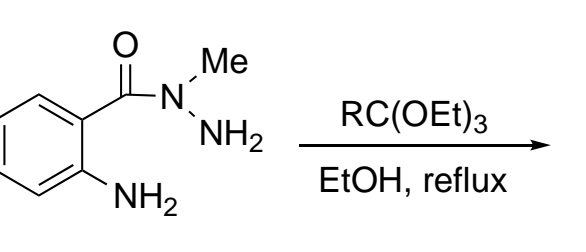<smiles>[R]C(=Nc1ccccc1C(=O)N(C)C)NN</smiles>

$\begin{aligned} \text { 200a }(R=H) & 46 \% \\ \text { b }(R=M e) & 57 \% \\ \text { c }(R=E t) & 58 \% \\ \text { d }(P=P h) & 63 \%\end{aligned}$

Scheme 56. Condensative closure of a seven-membered ring.

\section{Oxygen Heterocycles}

Warren and co-workers advanced an interesting synthesis of highly functionalized tetrahydrofurans (THF) and tetrahydropyrans (THP) involving an acid-catalyzed reaction of a series of triols with trimethyl orthoacetate (Scheme 57). ${ }^{98}$ The Sharpless asymmetric dihydroxylation was used to fashion a series of enantiomerically enriched 1,2,4-triols such as 201. Exposure of triol 201 to trimethyl orthoacetate and pyridinium $p$ toluenesulfonate (PPTS, $25 \mathrm{~mol} \%$ ) in DCM for $2 \mathrm{~min}$ resulted in quantitative conversion to the bicyclic orthoester 202. Extending the reaction time to $24 \mathrm{~h}$ with this mild acid catalyst, however, resulted in further conversion to a 23:73 mixture of the rearranged THF 203 and THP 204 derivatives in 98\% yield. These products derived from the two possible ring openings of a bridged episulfonium ion intermediate formed when a phenylthioether substituent assisted in the ring opening of an adjacent 5,6-dihydro-4H-1,3-dioxin-1ium ion. Further treatment of this mixture with $p-\mathrm{TsOH}$ in the same solvent resulted in isolation of THP 204 as the exclusive product (91\%). Following several control experiments, it was concluded that the THF compound was the kinetic product, while the THP was the thermodynamic product. A number of related triols were investigated $^{99}$ with similar, although slightly varying, outcomes. 


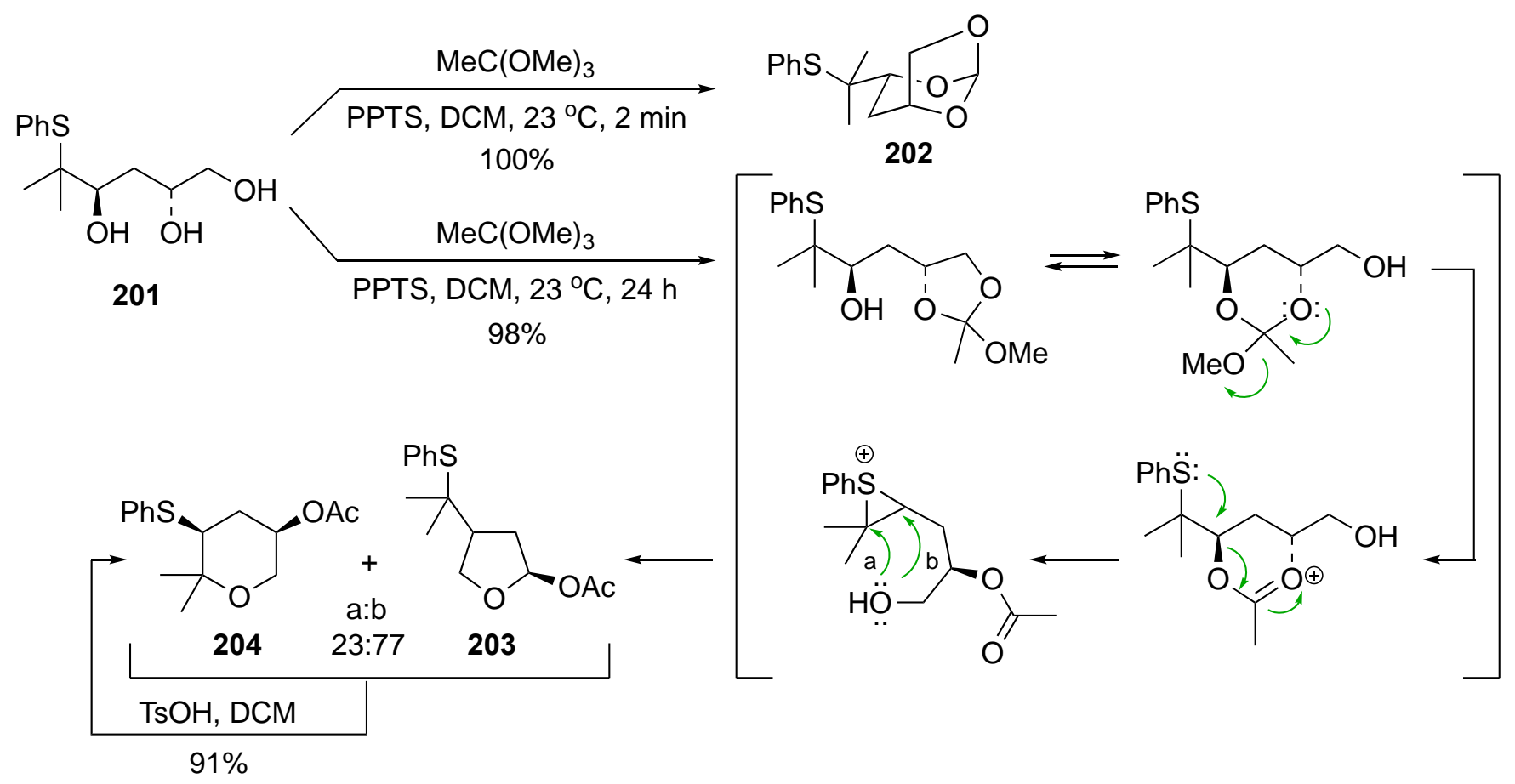

Scheme 57. An approach to highly functionalized tetrahydrofurans and tetrahydropyrans.

Clarke and co-workers have recently used orthoesters in a synthesis of dihydropyran-4-ones (Scheme 58). ${ }^{100}$ Inspired by the classic Maitland-Japp reaction, ${ }^{101} 5$-hydroxy- $\beta$-ketoesters were converted to the oxygen heterocycles by reaction with various orthoesters. For example, treatment of methyl 6-benzyloxy-5-hydroxy3-oxohexanoate (205, 1 equiv) with trimethyl orthovalerate ( 2 equiv) in the presence of acetic anhydride $\left(\mathrm{Ac}_{2} \mathrm{O}, 3.3\right.$ equiv) in toluene under $\mathrm{MW}$ heating $\left(150 \mathrm{~W}, 110{ }^{\circ} \mathrm{C}, 15 \mathrm{~min}\right)$ proceeded via 206 to give methyl 2((benzyloxy)methyl)-6-butyl-4-oxo-3,4-dihydro-2H-pyran-5-carboxylate (207) in 70\% yield. Alternatively, the conversion of $\mathbf{2 0 5}$ to $\mathbf{2 0 7}$ was also performed in refluxing toluene to give the dihydropyrans, although a large excess of the orthoester (10 equiv) was required, the reaction time was significantly longer, and the product was more difficult to purify.

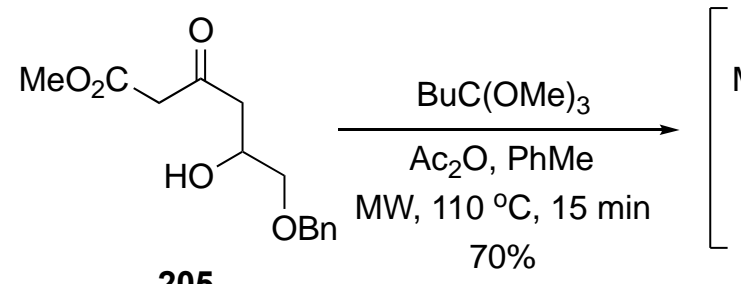

205

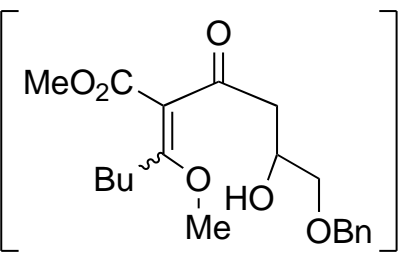

206

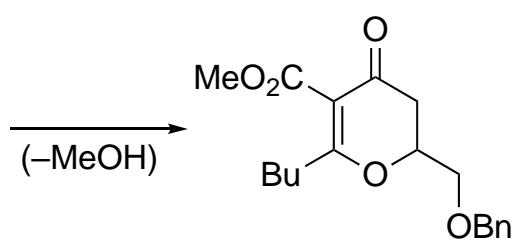

207

Scheme 58. Synthesis of dihydropyran-4-ones using orthoesters.

A novel preparation of five- and six-membered lactones has recently been reported from 2-(3chloropropyl)-1,3-dioxolane (208). ${ }^{102}$ These orthoesters are readily available from the nitriles via the Pinner synthesis. $^{30,103-104}$ Lithium halogen exchange with lithium powder catalyzed by 4,4'-(di-tert-butyl)biphenyl (DTBB, $5 \mathrm{~mol} \%$ ) in the presence of an aldehyde under Barbier-type conditions at $-78{ }^{\circ} \mathrm{C}$, followed by hydrolysis with phosphate buffer, gave 209. Sequential treatment with potassium hydrogen sulfate in 1,2dimethoxyethane:water (5:1), followed by lithium hydroxide to basic $\mathrm{pH}$, and finally refluxing in benzene 
containing catalytic $p$-TsOH yielded the six-ring lactones $\mathbf{2 1 0}$ in 33-47\% yield. As the lower 2-(2-chloroethyl)1,3-dioxolane homolog could not be prepared in pure form, it was not useful in generating five-membered lactones. However, butyrolactones were successfully prepared from 1-(2-chloroethyl)-2,6,7trioxabicyclo[2.2.2] octane (211) in 37-45\% yields (Scheme 59).
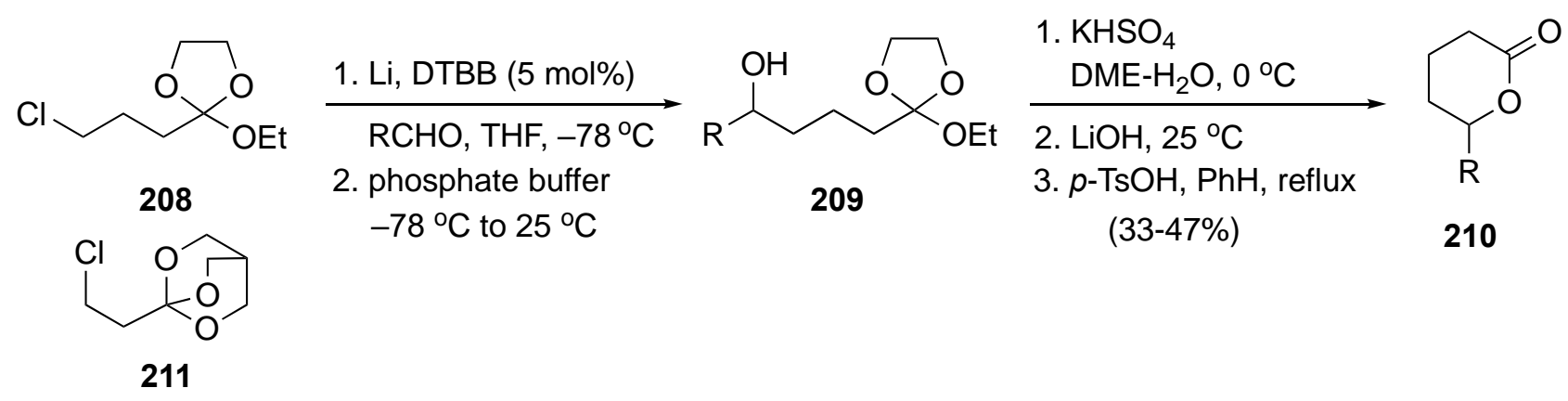

Scheme 59. Synthesis of five and six-membered lactones using orthoesters.

\section{Conclusions}

The research cited above clearly establishes the high potential of orthoesters in heterocycle synthesis. Since most of the studies delineated have employed simple commercial orthoester derivatives, it is clear that only the surface of this field has been explored. Future work should strive to prepare and evaluate new orthoester derivatives with additional functionality on the carbon group bound to the central oxygenated carbon. This would permit additional substitution patterns on the basic ring structures and invite further transformations of the initially formed products. These advancements would assure an expanding variety of new drug candidates and other derivatives for natural product and materials synthesis.

\section{Acknowledgements}

The author wishes to thank his students and collaborators, past and present, who have contributed ideas, expertise and hard work to make his research program successful. He is also grateful to funding agencies (NIH, NSF, ACS-PRF, Research Corporation), the State of Oklahoma, Oklahoma State University and the OSU Chemistry Department for providing funding and facilities to allow a continuous research effort, as well as scholarships and assistantships to help support his students. Finally, the author wishes to express his appreciation to the library team at the Edmon Low Library at OSU for helping to provide access to many of the journal articles cited in this review.

\section{References}

1. McElvain, S. M.; Nelson, J. W. J. Am. Chem. Soc., 1942, 64, 1825. https://doi.org/10.1021/ja01260a024

2. Lebel, H.; Grenon, M. Sci. Synth. 2005, 22, 669. 
3. Kacem, Y.; Hassine, B. B. Heterocycles 2014, 89, 197.

doi:10.3987/COM-13-12878

4. von Rauch, M.; Schlenk, M.; Gust, R. J. Med. Chem. 2004, 47, 915.

https://doi.org/10.1021/jm0309809

5. Lee, J. J.; Kim, J.; Jun, Y. M.; Lee, B. M.; Kim, B. H. Tetrahedron 2009, 65, 8821.

https://doi.org/10.1016/j.tet.2009.08.059

6. Bel'skaya, N. P.; Koksharov, A. V.; Lesogorova, S. G.; Slepukhin, P. A. ; Bakulev. V. A. Russ. Chem. Bull. 2011,

$60,889$.

https://doi.org/10.1007/s11172-011-0139-2

7. Katritzky, A. R.; Ramsden, C. A.; Joule, J. A.; Zhdankin, V. V. Handbook of Heterocyclic Chemistry, 3rd Ed.; Elsevier, Amsterdam, 2010; pp. 775-777.

8. Polshettiwar, V.; Varma, R. S. Tetrahedron Lett. 2008, 49, 879.

https://doi.org/10.1016/i.tetlet.2007.11.165

9. Polshettiwar, V; Kaushik, M. P. Tetrahedron Lett. 2004, 45, 6255.

https://doi.org/10.1016/j.tetlet.2004.06.091

10. Gnanasekaran, K. K.; Nammalwar, B.; Murie, M.; Bunce, R. A. Tetrahedron Lett. 2014, 55, 6776. https://doi.org/10.1016/j.tetlet.2014.10.028

11. Kudelko, A.; Jasiak, K. Synthesis 2013, 45, 1950.

https://doi.10.1055/s-0033-1338454

12. Kudelko, A. Tetrahedron 2012, 69, 3616.

https://doi.org/10.1016/i.tet.2012.02.071

13. Zielinski, W.; Kudelko, A.; Czardybon, W. J. Heterocycl. Chem. 2005, 42, 1393.

https://doi.org/10.1002/jhet.5570420720

14. Kudelko, A. Tetrahedron 2011, 68, 8502.

https://doi.org/10.1016/j.tet.2011.09.018

15. Kudelko, A.; Zielinski, W. Tetrahedron 2009, 65, 1200.

https://doi.org/10.1016/j.tet.2008.11.096

16. Toche, R. B.; Janrao, R. A. ; Gangurde, S. A.; Nikam, P. S. Synthetic Commun. 2013, 43, 2527. https://doi.org/10.1080/00397911.2012.719256

17. Katritzky, A. R.; Ramsden, C. A.; Joule, J. A.; Zhdankin, V. V. Handbook of Heterocyclic Chemistry, 3rd Ed.; Elsevier, Amsterdam, 2010; pp. 792-793.

18. Aridoss, G.; Laali, K. K. Eur. J. Org. Chem. 2011, 2827.

https://doi.org/10.1002/ejoc.201100128

19. Grigoriev, Y. V.; Voitekhovich, S. V.; Karavai, V. P.; Ivashkevich, O. A. Chem. Heterocycl. Compd. 2017, $53,670$.

https://doi.org/10.1007/s10593-017-2108-7

20. Katritzky, A. R.; Ramsden, C. A.; Joule, J. A.; Zhdankin, V. V. Handbook of Heterocyclic Chemistry, 3rd Ed.; Elsevier, Amsterdam, 2010; pp. 836-838.

21. Jenkins, G. L.; Knevel, A. M.; Davis, C. S. J. Org. Chem. 1961, 26, 274.

https://doi.org/10.1021/jo01060a626

22. Kaboudin, B.; Khodamorady, M.; Abedi, Y. Org. Prep. Proced. Int. 2013, 45, 162. https://doi.org/10.1080/00304948.2013.765294

23. Villemin, D.; Hammadi, M.; Martin, B. Synthetic Commun. 1996, 26, 2895. 
https://doi.org/10.1080/00397919608005224

24. Fortenberry, C.; Nammalwar, B.; Bunce, R. A. Org. Prep. Proced. Int. 2013, 45, 57. https://doi.org/10.1080/00304948.2013.743751

25. Mike, J. F.; Inteman, J. J.; Ellern, A.; Jeffries-El, M. J. Org. Chem. 2010, 75, 495. https://doi.org/10.1021/jo9023864

26. Hojati, S. F.; Maleki, B.; Beykzadeh, Z. Monatsh. Chem. 2011, 142, 87. https://doi.org/10.1007/s00706-010-0412-3

27. Khaksar, S.; Heydari, A.; Tajbakhsh, M.; Vahdat, S. M. J. Fluorine Chem. 2010, 131, 1377. http://dx.doi.org/10.1016/j.jfluchem.2010.10.002

28. Srivani, A.; Venkateswar Rao, K. T.; Sai Prasad, P. S.; Lingaiah, N. J. Mol. Cat. A: Chem. 2010, $328,119$. https://doi.org/10.1016/j.molcata.2010.06.009

29. Montazeri, N.; Rad-Moghadam, K. Asian. J. Chem. 2006, 18, 1557. www.asianjournalofchemistry.co.in/User/ViewFreeArticle.aspx?ArticleID=18 2132

30. Bastug, G.; Eviolitte, C.; Marko, I. E. Org. Lett. 2012, 14, 3502. https://doi.org/10.1021/ol301472a

31. Vargas-Oviedo, D.; Charris-Molina, A.; Portilla, J. ChemistrySelect, 2017, 2, 3896. https://doi.org/10.1002/slct.201700623

32. Liu, Z.; Li, H.; Zhao, Q.; Shen, J. Heterocycles 2008, 75, 1907. doi: 10.3987/COM-08-11384

33. Chorvat, R. J.; Bakthavatchalam, R.; Beck, J. P.; Gilligan, P. J.; Wilde, R. G.; Cocuzza, A. J.; Hobbs, F. W.; Cheeseman, R. S.; Curry, M.; Resinito, J. P.; Krenitsky, P.; Chidester, D.; Yarem, J. A.; Klaczkiewicz, J. D.; Hodge, C. N.; Aldrich, P. E.; Wasserman, Z. R.; Fernandez, C. H.; Zaczek, R.; Fitzgerald, L. W.; Huang, S.-M.; Shen, H. L.; Wong, N.; Chien, B. M.; Quon, C. Y.; Arvanitis, A. J. Med. Chem. 1999, 42, 833. https://doi.org/10.1021/jm980224g

34. Hammarström, L. G. J.; Smith, D. B.; Talamás, F. X. Tetrahedron Lett. 2007, 48, 2823. https://doi.org/10.1016/j.tetlet.2007.02.104

35. Castillo, J.-C.; Estupiñan, D.; Nogueras, M.; Cobo, J.; Portilla, J. J. Org. Chem. 2016, 81, 12364. https://doi.org/10.1021/acs.joc.6b02431

36. McQuaid, L. A.; Smith E. C. R.; South, K. K.; Mitch, C. H.; Schoepp, D. D.; True, R. A.; Calligaro, D. O.; O'Malley, P. J.; Lodge, D. Ornstein, P. L. J. Med. Chem. 1992, 35, 3319.

https://doi.org/10.1021/jm00096a002

37. Dreikorn, B. A.; Thibault, T. D. U.S. Patent 4,008,322, 1977; Chem. Abstr. 1977, 86, 166387.

38. Makino, K; Sakata, G.; Morimoto, K.; Ochiai, Y. Heterocycles 1985, 23, 2025. doi: 10.3987/R-1985-08-2025

39. Ribeill, Y.; Genevois-Borella, A.; Vuilhorgne, M.; Mignani, S. Heterocycles 1999, 51, 2977. doi: 10.3987/COM-99-8682

40. Hsu, W.-C.; Hwang, J.-M.; Hsu, L.-Y.; Huang, Y.-Y.; Liu, K.-C. Chin Pharm. J. (Taipei, Taiwan) 2003, $55,185$.

http://www.speciation.net/Database/Journals/The-Chinese-pharmaceutical-journal-;i1047

41. Davoodnia, A.; Saeidi, A.; Tavakoli-Hoseini, N. Asian J. Chem. 2012, 24, 2313. www.asianjournalofchemistry.co.in/User/ViewFreeArticle.aspx?ArticleID=24 $5 \quad 104$

42. Davoodnia, A.; Zhiani, R.; Roshani, M.; Bakavoli, M.; Bashash, M. Phosphorus, Sulfur, Silicon 2007, 182, 1219. 
43. Davoodnia, A.; Zhiani, R.; Tavakoli-Hoseini, N. Monatsh. Chem. 2008, 139, 1405. https://doi.org/10.1007/s00706-008-0939-8

44. Sheikhi-Mohammareh, S.; Shiri, A.; Bakavoli, N. J. Chem. Res. 2015, 39, 403. https://doi.org/10.3184/174751915X14357589569981

45. Davoodnia, A.; Bakavoli, M.; Mohseni, S.; Tavakoli-Hoseini, N. Monatsh. Chem. 2008, 139, 963. https://doi.org/10.1007/s00706-007-0844-6

46. Moradi, H.; Eshghi, H.; Bakavoli, M. J. Chem. Res. 2016, 40, 276. https://doi.org/10.3184/174751916X14598638014154

47. Montazeri, N.; Heravi, M. M.; Tajfirooz, F.; Nazari, N. Monatsh. Chem. 2017, 148, 691. https://doi.org/10.1007/s00706-016-1810-y

48. Kulikov, A. S.; Epishina, M. A.; Fershtat, L. L.; Makhova, N. N. Chem. Heterocycl. Compd. 2018, 54, 669. https://doi.org/10.1007/s10593-018-2325-8

49. Kürti, L.; Czakó, B. Strategic Applications of Named Reaction in Organic Synthesis; Elsevier: New York, NY; 2005; p. 144.

50. Brown, D. J. Mech. Mol. Migr. 1968, 1, 209.

51. Potts, K. T.; Burton, H. R. Proc. Chem. Soc. 1964, 420. https://doi.org/10.1039/PS9640000385

52. Paudler, W. W.; Helmick, L. S. J. Heterocycl. Chem. 1966, 3, 269. https://doi.org/10.1002/jhet.5570030306

53. Rousseaux, O.; Blondeau, D.; Sliwa, H. Tetrahedron Lett. 1986, 27, 3127. https://doi.org/10.1016/S0040-4039(00)84732-2

54. Brown, D. J.; Shinozuka, K. Aust. J. Chem. 1981, 34, 189. https://doi.org/10.1071/CH9810189

55. Nagamatsu, T.; Fujita, T. Heterocycles 2002, 57, 631. doi: 10.3987/COM-02-9450

56. Hsu, W.-C.; Tao, T.-M.; Chou, T.-C.; Ding, Y.-A.; Liu, K.-C. Chin. Pharm. J. (Taipei, Taiwan) 2002, 54, 331.

http://www.speciation.net/Database/Journals/The-Chinese-pharmaceutical-journal-;i1047

57. Sakai, N.; Aoki, Y.; Sasada, T.; Konakahara, T. Org. Lett. 2005, 7, 4705. https://doi.org/10.1021/ol051901l

58. Sasada, T.; Aoki, Y.; Ikeda, R.; Sakai, N.; Konakahara, T. Chem Eur. J. 2011, 17, 9385. https://doi.org/10.1002/chem.201100040

59. Butler, K.; Partridge, M. W.; Waite, J. A. J. Chem. Soc. 1960, 4970. https://doi.org/10.1039/JR9600004970

60. Stephen, T.; Stephen, H. J. Chem. Soc. 1956, 4178. https://doi.org/10.1039/JR9560004178

61. Partridge, M. W.; Slorach, S. A.; Vipond, H. J. J. Chem. Soc. 1964, 3670. https://doi.org/10.1039/JR9640003670

62. Gavin, J. T.; Annor-Gyamfi, J. K.; Bunce, R. A. Molecules 2018, 23, 2925. https://doi.org/10.3390/molecules23112925

63. El-Gaby, M. S. A.; El-Dean, A. M. K.; Gaber, A. E. M.; Eyada, H. A.; Al-Kamali, A. S. N. Bull. Korean Chem. Soc.

2003, 24, 1181.

https://doi.org/10.5012/bkcs.2003.24.8.1181 
64. Druey, J.; Schmidt, P. Swiss Patent CH 320131, 1957; Chem. Abstr. 1958, 52, 35530.

65. Hazarkhani, H.; Karimi, B. Tetrahedron 2003, 59, 4757. https://doi.org/10.1016/S0040-4020(03)00696-3

66. Davoodnia, A. Asian J. Chem. 2010, 22, 1591. http://www.asianjournalofchemistry.co.in/user/journal/viewarticle.aspx?ArticlelD=22 2110

67. Davoodnia, A.; Bakavoli, M.; Barakouhi, Gh.; Tavakoli-Hoseini, N. Chin Chem. Lett. 2007, $18,1483$. https://doi.org/10.1016/j.cclet.2007.10.013

68. Takavoli-Hoseini, N.; Moloudi, R.; Davoodnia, A.; Shaker, M. Chin. J. Chem. 2011, 29, 2421. https://doi.org/10.1002/cjoc.201180411

69. Davoodnia, A.; Moloudi, R.; Takavoli-Hoseini, N.; Shaker, M. Asian J. Chem. 2012, 24, 2195. http://www.asianjournalofchemistry.co.in/user/journal/viewarticle.aspx?ArticlelD=24_5 74

70. Okamoto, Y.; Yamaguchi, Y.; Kurasawa, Y.; Maeda, M. J. Heterocycl. Chem. 2008, 45, 717. https://doi.org/10.1002/jhet.5570450313

71. Shi, D.; Chi, C.; Wang, J.; Rong, L.; Zhuang, Q.; Wang, X. J. Heterocycl. Chem. 2005, 42, 173. https://doi.org/10.1002/jhet.5570420201

72. Agrebi, A.; Allouche, F.; Fetoui, H.; Chabchoub, F. Mediterranean J. Chem. 2014, 3, 864. http://dx.doi.org/10.13171/mjc.3.2.2014.13.05.23

73. Davoodnia, A.; Rshani, M.; Malaeke, S. H.; Bakavoli, M. Chin Chem. Lett. 2008, 19, 525. https://doi.org/10.1016/j.cclet.2008.01.037

74. Davoodnia, A.; Bakavoli, M.; Elmi-Mehr, M. Indian J. Heterocycl. Chem. 2008, 17, 371. https://www.researchgate.net/publication/289256325

75. Annor-Gyamfi, J. K.; Bunce, R. A. Molecules 2019, 24, 3555. https://doi.org/10.3390/molecules24193555

76. Bel'skaya, N. P.; Zvereva, E. E.; Babushkina, L. A.; Bakulev, V. A. Chem. Heterocycl. Compd. 2000, 36, 1066.

https://doi.org/10.1023/A:1002738100046

77. Fujii, T.; Itaya, T.; Mohri, K.; Saito, T. J. Chem. Soc., Chem. Commun. 1973, 917. https://doi.org/10.1039/C3973000917A

78. Fujii, T.; Itaya, T. Tetrahedron, 1971, 27, 351. https://doi.org/10.1016/S0040-4020(01)90704-5

79. Piskala, A.; Cihak, A; Korbova, L. Czech Patent CS 269077, 1990; Chem. Abstr. 1992, 114, 229310.

80. Zemanová, I.; Gasparová, R.; Kraic, F.; Kruslicová, D.; Maliar, T.; Bohác, A.; Addová, G. Arkivoc 2017, iv, 184.

https://doi.org/10.24820/ark.5550190.p009.788

81. Krutosíková, A.; Kovác, J.; Dandárová, M. Collect. Czech. Chem. Commun. 1984, 49, 65. https://doi.org/10.1135/cccc19840065

82. Atkinson, R. S.; Kelly, B. J.; Williams, J. Tetrahedron 1992, 48, 7713. https://doi.org/10.1016/S0040-4020(01)90382-5

83. Coogan, M. P.; Ooi, L-L.; Pertusati, F. Org. Biomol. Chem. 2005, 3, 1134. https://doi.org/10.1039/B419108K

84. Fischer, M.; Troschütz, R. Synthesis 2003, 1603. doi: $10.1055 / \mathrm{s}-2003-40524$

85. Haake, M.; Schümmelfeder, B. Synthesis 1991, 753. 
86. Heravi, M. M.; Bakavoli, M.; Rahimizadeh, M.; Rajabzadeh, G.; Ghassemzadeh, M. Russ. J. Org. Chem. 2008,

44, 1233.

https://doi.org/10.1134/S1070428008080216

87. Khan, I. A.; Kulkarni, M. V.; Gopal, M.; Shahabuddin, M. S.; Sun, C.-M. Bioorg. Med. Chem. Lett. 2005, $15,3584$.

https://doi.org/10.1016/j.bmcl.2005.05.063

88. Mkaouar, K.; Chabchoub, F.; Samadi, A.; Contelles, J. L. M.; Salem, M. Synth. Commun. 2010, 40, 3405. https://doi.org/10.1080/00397910903419928

89. Chaker, A.; Najahi, E.; Nepveu, F.; Chabchoub, F. Arabian J. Chem. 2013, 10, S3040.

https://doi.org/10.1016/j.arabjc.2013.11.045

90. Ghotekar, B. K.; Jachak, M. N.; Toche, R.B. J. Heterocycl. Chem. 2009, 46, 708.

https://doi.org/10.1002/jhet.128

91. Khashi, M.; Davoodnia, A.; Prasada Rao Lingam, V. S. Res. Chem. Intermed. 2015, 41, 5731

https://doi.org/10.1007/s11164-014-1697-3

92. Al-Azmi, A.; Booth, B. L.; Carpenter, R. A.; Carvalho, A.; Marrelec, E.; Pritchard, R. G.; Proença, M. F. J.

R. P. J. Chem. Soc., Perkin Trans. 1 2001, 2532.

https://doi.org/10.1039/B106539B

93. Al-Azmi, A.; Kumari, K. A. Heterocycles, 2009, 78, 2951.

doi: 10.3987/COM-09-11794

94. Zaki, M. E. A.; Proença, M. F.; Booth, B. L. J. Org. Chem. 2003, 68, 276.

https://doi.org/10.1021/jo020347f

95. Al-Azmi, A.; Kumari, K. A. Heterocycles 2009, 78, 2245.

doi: 10.3987/COM-09-11713

96. Derstine, B. P.; Tomlin, J. W.; Peck, C. L.; Dietz, J.-P.; Herrera, B. T.; Cardoso, F. S. P.; Paymode, D. J.; Yue, A. C.; Arduengo, A. J. III; Opatz, T.; Snead, D. R.; Stringham, R. W.; McQuade, D. T.; Gupton, B. F. Org. Process Res. Dev. 2020, 24, 1420-1427.

https://doi.org/10.1021/acs.oprd.0c00078

97. Sunder, S.; Peet, N. P.; Trepanier, D. L. J. Org. Chem. 1976, 41, 2732.

https://doi.org/10.1021/jo00878a019

98. Caggiano, L.; Fox, D. J.; House, D.; Jones, Z. A.; Kerr, F.; Warren, S. J. Chem. Soc., Perkin Trans 1 2002, 2646.

https://doi.org/10.1039/B208557G

99. House, D.; Kerr, F.; Warren, S. J. Chem. Soc., Perkin Trans. 1 2002, 2652.

https://doi.org/10.1039/B208558E

100. Clarke, P. A.; Sellars, P. B.; Nasir, N. M. Org. Biomol. Chem. 2015, 13, 4743.

https://doi.org/10.1039/C50B00292C

101. Japp, F. R.; Maitland, W. J. Chem Soc. 1904, 85, 1473.

https://doi.org/10.1039/СT9048501473

102. Yus, M.; Torregrosa, R.; Pastor, I. M. Molecules 2004, 9, 330.

https://doi.org/10.3390/90500330

103. DeWolfe, R. H. Synthesis 1974, 153.

doi: $10.1055 / \mathrm{s}-1974-23268$

104. Casy, G.; Patterson, J. W.; Taylor, R. J. K. Org. Synth. 1989, 67, 193. 
doi:10.15227/orgsyn.067.0193

105. Ueno, H.; Maruyama, A.; Miyake, M.; Nakao, E.; Nakao, K.; Umezu, K.; Nitta, I. J. Med. Chem. 1991, 34, 2468.

https://doi.org/10.1021/im00112a023

\section{Author Biography}

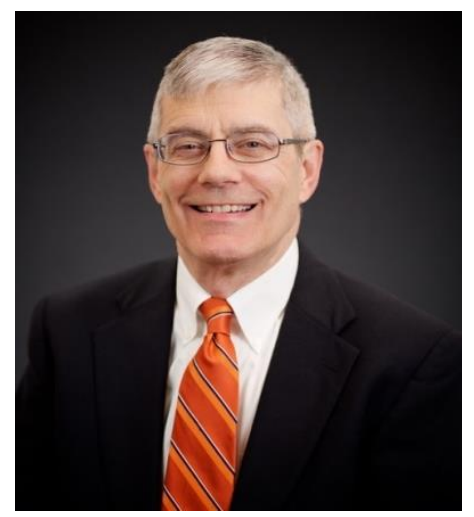

Richard A. Bunce was born and raised in Connecticut. He earned his B.S. degree in Chemistry from Marietta College (Marietta, Ohio) in 1975 and his Ph.D. in Organic Chemistry from the University of WisconsinMadison in 1981. From 1981-1983, he was a National Institutes of Health Postdoctoral Fellow at the University of California-Berkeley. He has been on the faculty at Oklahoma State University since 1983 and was recently named a Regents Professor. His research interests include mechanistic organic chemistry, the development of new synthetic approaches to heterocyclic compounds, and the synthesis of medicinal agents. 